\title{
Teitelbaum's exceptional zero conjecture in the anticyclotomic setting
}

\author{
Massimo Bertolini \\ Henri Darmon \\ Adrian Iovita \\ Michael Spiess
}

September 5, 2007

\section{Contents}

1 Preliminaries 5

1.1 Review ...................... 5

1.2 Harmonic cocycles . . . . . . . . . . . . . . . . . 9

1.3 Schneider's distribution . . . . . . . . . . . . . . . 13

2 The $p$-adic $L$-function $\quad 15$

2.1 Complex $L$-functions . . . . . . . . . . . . . . . . . . . . . . . 16

2.2 The basic strategy . . . . . . . . . . . . . . . . . . 17

2.3 Embeddings of $K$ into $B \ldots \ldots \ldots \ldots \ldots$

2.4 The $p$-adic distribution attached to an embedding . . . . . . 20

2.5 Interpolation of classical special values . . . . . . . . . . . . 26

2.6 The $p$-adic $L$-function . . . . . . . . . . . . . . . . . . . . . . . 35

3 Proof of the main identities $\quad 38$

3.1 Teitelbaum's $\mathcal{L}$-invariant . . . . . . . . . . . . . . . . . . 38

3.2 The split case . . . . . . . . . . . . . . . . . . . . . . . 39

3.3 The inert case . . . . . . . . . . . . . . . . 43 


\begin{abstract}
In [Tei], Teitelbaum formulates a conjecture relating first derivatives of the MazurSwinnerton-Dyer $p$-adic $L$-functions attached to a modular forms of even weight $k \geq 2$ to certain $\mathcal{L}$-invariants arising from Shimura curve parametrisations. This article formulates an analogue of Teitelbaum's conjecture in which the cyclotomic $\mathbb{Z}_{p}$ extension of $\mathbb{Q}$ is replaced by the anticyclotomic $\mathbb{Z}_{p}$-extension of an imaginary quadratic field. This analogue is then proved by using the Cerednik-Drinfeld theory of $p$-adic uniformisation of Shimura curves.
\end{abstract}

\title{
Introduction
}

Let $\phi=\sum a_{n} q^{n}$ be an eigenform of even weight $k \geq 2$ on $\Gamma_{0}(N)$. The classical $L$-function $L(\phi, s)$ admits an analytic continuation to the entire complex plane, and a functional equation which relates its values at $s$ and $k-s$. Of special arithmetic interest for the present work is the central value $L(\phi, k / 2)$.

For example, when $k=2$, the Birch and Swinnerton-Dyer conjecture relates the behaviour of $L(\phi, s)$ at $s=1$ to the arithmetic of the abelian variety $A_{\phi}$ associated to $\phi$ by the Eichler-Shimura construction. In [MTT], a $p$-adic variant of the Birch and Swinnerton-Dyer conjecture is formulated with $L(\phi, s)$ replaced by a $p$-adic analogue $L_{p}(\phi, s)$ attached to the cyclotomic $\mathbb{Z}_{p}$-extension of $\mathbb{Q}$.

When $p$ divides $N$ exactly and $a_{p}=1$ (which implies that $A_{\phi}$ has split multiplicative reduction at $p)$, the function $L_{p}(\phi, s)$ vanishes at $s=1$. In this case the conjectures of [MTT] imply the following relationship between the first derivative $L_{p}^{\prime}(\phi, 1)$ and the special value $L(\phi, 1)$ :

$$
L_{p}^{\prime}(\phi, 1)=\mathcal{L}(\phi) \cdot L(\phi, 1) / \Omega,
$$

where $\Omega$ is an appropriate real period and $\mathcal{L}(\phi)$, the so-called $\mathcal{L}$-invariant, is an isogeny invariant of $A_{\phi / \mathbb{Q}_{p}}$. It is defined by using the Tate-Morikawa theory of p-adic uniformisation of abelian varieties with multiplicative reduction at $p$. For example, when $\phi$ has rational Fourier coefficients, so that $A_{\phi}$ is an elliptic curve, then

$$
\mathcal{L}(\phi)=\frac{\log _{p}(q)}{\operatorname{ord}_{p}(q)}
$$


where $q \in \mathbb{Q}_{p}^{\times}$is the Tate period associated to $A_{\phi}$ over $\mathbb{Q}_{p}$, and $\log _{p}$ is the branch of the $p$-adic $\operatorname{logarithm}$ such that $\log p=0$. The conjectured relation (1) was proved by Greenberg and Stevens [GS] using Hida's theory of $p$ adic families of ordinary eigenforms and the two-variable $p$-adic $L$-functions attached to them.

Subsequently, much work has gone into formulating and proving similar conjectures for modular forms of higher (even) weight. The following generalisation of (1) was conjectured in this setting,

$$
L_{p}^{\prime}(\phi, k / 2)=\mathcal{L}(\phi) \cdot L(\phi, k / 2) / \Omega,
$$

with several possible definitions for the $\mathcal{L}$-invariant.

1. The first candidate for $\mathcal{L}(\phi)$, denoted $\mathcal{L}_{T}(\phi)$, was proposed by Teitelbaum [Tei], relying on the Jacquet-Langlands correspondence between forms on $\Gamma_{0}(N)$ and on certain indefinite quaternion algebras, and on the CerednikDrinfeld theory of $p$-adic uniformisation of Shimura curves. In particular, $\mathcal{L}_{T}(\phi)$ is only defined when $\phi$ can be associated via the Jacquet-Langlands correspondence to a modular form on such a Shimura curve.

2. A second invariant $\mathcal{L}_{C}(\phi)$ was proposed by Coleman [Co], based on his theory of $p$-adic integration on the modular curve $X_{0}(N)$.

3. Finally, a third $\mathcal{L}$-invariant $\mathcal{L}_{F M}(\phi)$ was introduced by Fontaine and Mazur [Mz1] in terms of the semistable Dieudonné module of the local $p$ adic Galois representation attached to $\phi$.

Proofs of equation (2) have been announced by Stevens and by Kato, Kurihara and Tsuji, with $\mathcal{L}(\phi)$ replaced by $\mathcal{L}_{C}(\phi)$ and $\mathcal{L}_{F M}(\phi)$ respectively.

Parallel to these developments, a $p$-adic conjecture of Birch and Swinnerton-Dyer type (for weight two modular forms) is formulated in [BD1], with the cyclotomic $\mathbb{Z}_{p}$-extension of $\mathbb{Q}$ replaced by the anticyclotomic $\mathbb{Z}_{p^{-}}$ extension of a quadratic imaginary field $K$. In the anticyclotomic setting, the $p$-adic $L$-function admits a construction which appeals to the $p$-adic analytic uniformization of Shimura curves, unlike the cyclotomic setting, where the complex uniformisation is required in the definition of modular symbols. Thus the anticyclotomic setting is more amenable to the purely $p$-adic approach initially proposed by [Sch] for the cyclotomic setting. Furthermore, new exceptional zero phenomena emerge which have no counterpart 
in the situation explored by Mazur, Tate and Teitelbaum, and which can be approached through other methods, most notably the theory of complex multiplication. (See for example the formulae of [BD2] and [BD3].)

When the prime $p$ splits in $K$, the article [BD4] gives a proof of formula (1) in the anticyclotomic context. This proof differs from the one given by Greenberg and Stevens in the cyclotomic case, making no use of Hida families or two-variable $p$-adic $L$-functions, but relying on $p$-adic integration on Shimura curves in an essential way. Since this ingredient is precisely the one that enters both into the definition of Teitelbaum's $\mathcal{L}$-invariant and of the anticyclotomic $p$-adic $L$-function, it is natural to build on the methods of [BD4] to prove the anticyclotomic analogue of Teitelbaum's conjecture. This is one task carried out in this paper. (Cf. theorem 3.4 of section 3.2.)

The case where $p$ is inert in $K$ (which for weight 2 is treated in [BD3]) is different: both the $p$-adic and classical $L$-functions vanish at the central point, and the discrepancy between them cannot be accounted for by a simple $\mathcal{L}$-invariant. This case is discussed in section 3.3; theorem 3.5 expresses $L_{p}^{\prime}(\phi, k / 2)$ in terms of a $p$-adic Coleman integral between two CM points on the $p$-adic upper half plane, and can be viewed as a generalisation of one of the main results of [BD3] to weight $k>2$. The arithmetic interpretation of this integral will be explored in a future work.

Note that by combining the above mentioned results of Stevens with those of Kato-Kurihara-Tsuji implies the equality of the (cyclotomic) Coleman and Fontaine-Mazur $\mathcal{L}$-invariants. In general, a direct, local comparison of these $\mathcal{L}$-invariants is investigated in [CI] where it is shown that they are equal when they are defined. This applies to the present work, so that the anticyclotomic analogue of $\mathcal{L}_{T}(\phi)$ could be replaced by $\mathcal{L}_{C}(\phi)$ or $\mathcal{L}_{F M}(\phi)$ in the statement of theorem 3.4.

The present work has its roots in a graduate course taught by one of the authors (Iovita) at McGill University in the Spring of 1998, in which the connection between the $p$-adic $L$-function of [BD1] and Schneider's rigid analytic $L$-transforms was emphasized. The first three authors were then led to recast the proofs of the main results of [BD3] and [BD4] in this framework, while a similar approach was being developed independently by the fourth author. What emerged were proofs of the main results of [BD3] and [BD4] which, while not fundamentally different from the originals, are more elegant and conceptual, and clarify the role of the underlying machinery of $p$-adic in- 
tegration. In particular, the use of Schneider's $p$-adic boundary distributions and Teitelbaum's $p$-adic Poisson transform, implicit in [BD3] and [BD4], is made explicit. This point of view has been influential in later work, notably [Da].

Because of the more conceptual point of view taken in the present work, even the reader interested only in the weight two results of [BD3] and [BD4] may find it helpful to study this paper after setting $k=2$. The authors also felt it would be worthwhile to generalize the results of [BD3] and [BD4] to higher weight, a context in which the original approach had seemed unduly burdensome to carry through.

Acknowledgements: It is a pleasure to thank Andreas Schweizer and Glenn Stevens for many inspiring discussions on quaternion algebras, $p$-adic integration and p-adic $L$-functions. Bertolini thanks the University of Strasbourg, Darmon the ETH (Zurich), and Iovita the University of Münster for their hospitality while much of this research was carried out. Two of the authors (Darmon and Iovita) were partially supported by CICMA (Centre Interuniversitaire en Calcul Mathématique Algébrique). Finally the authors thank the referee for helpful suggestions which led to significant improvements in the article.

\section{Preliminaries}

\subsection{Review}

In this section we review the main ingredients which enter our constructions and fix the notations.

The $p$-adic upper half plane. Let $\mathbb{C}_{p}$ be the completion of the algebraic closure $\overline{\mathbb{Q}}_{p}$ of $\mathbb{Q}_{p}$, and let

$$
\mathcal{H}_{p}:=\mathbb{P}_{1}\left(\mathbb{C}_{p}\right)-\mathbb{P}_{1}\left(\mathbb{Q}_{p}\right)
$$

be Drinfeld's $p$-adic upper half plane. It has a natural structure of a rigid analytic space (see [SS] where a more general situation is treated or [BD5] $\S 3.1$ for details on what is need here and where the notations are consistent with the ones in the current paper.) The group $\mathbf{P G L}_{2}\left(\mathbb{Q}_{p}\right)$ acts on $\mathcal{H}_{p}$ by 
fractional linear transformations. Fix once and for all an embedding of $\overline{\mathbb{Q}}$ into $\overline{\mathbb{Q}}_{p}$, and hence $\mathbb{C}_{p}$.

Write $\mathcal{T}=\mathcal{T}_{p}$ for the Bruhat-Tits tree of $\mathbf{P G L}_{2}\left(\mathbb{Q}_{p}\right)$ and denote by $\overrightarrow{\mathcal{E}}(\mathcal{T})$ the set of its oriented edges. If $e \in \overrightarrow{\mathcal{E}}(\mathcal{T})$ we denote by $V(e)$ the inverse image under reduction of $e$. It is a wide open annulus in $\mathcal{H}_{p}$.

Quaternion algebras. Let $\mathcal{B}$ be an indefinite quaternion algebra over $\mathbb{Q}$, i.e., a central simple algebra of rank 4 satisfying

$$
\mathcal{B} \otimes_{\mathbb{Q}} \mathbb{R} \simeq M_{2}(\mathbb{R}) .
$$

An order in $\mathcal{B}$ is a subring of $\mathcal{B}$ which is of rank 4 as a $\mathbb{Z}$-module. A maximal order is an order which is contained in no larger order, and an Eichler order is the intersection of two maximal orders. (For the definition of the level of an Eichler order, see [Vi], ch. I, §4.)

Let $B$ be a definite quaternion algebra, i.e., a quaternion algebra over $\mathbb{Q}$ satisfying

$$
B \otimes_{\mathbb{Q}} \mathbb{R} \simeq \mathbb{H},
$$

where $\mathbb{H}=\mathbb{R}+\mathbb{R} i+\mathbb{R} j+\mathbb{R} k$ is Hamilton's skew field of real quaternions. The algebra $B$ does not satisfy the Eichler condition, and in general contains several distinct conjugacy classes of maximal orders. (The number of such classes is called the type number of $B$, cf. [Vi], ch. V.)

Fix a prime $p$ for which $B$ splits. A $\mathbb{Z}[1 / p]$-order in $B$ is a subring of $B$ which is stable under multiplication by $\mathbb{Z}[1 / p]$ and is of rank 4 as a $\mathbb{Z}[1 / p]$ module. A maximal $\mathbb{Z}[1 / p]$-order of $B$ is a $\mathbb{Z}[1 / p]$-order which is contained in no larger $\mathbb{Z}[1 / p]$-order, and an Eichler $\mathbb{Z}[1 / p]$-order is the intersection of two maximal $\mathbb{Z}[1 / p]$-orders. For more details on quaternion algebras see [BD5] $\S 3.2$.

Shimura curves. Let $S$ be a finite set of places of $\mathbb{Q}$ of odd cardinality containing the place $\infty$, and let $N^{+}$be an integer which is not divisible by any prime in $S$. Then a Shimura curve $X$ over $\mathbb{Q}$ can be associated to the data $\left(S, N^{+}\right)$and described as follows. The presentation of the material is inspired by [Gr1], ch.IV.

Let $\mathcal{B}$ be the indefinite quaternion algebra ramified exactly at the places in $S-\{\infty\}$, let $\mathcal{R}$ be an Eichler order in $\mathcal{B}$ of level $N^{+}$, and let $\mathcal{R}^{\max }$ be a maximal order containing $\mathcal{R}$. Let $\mathcal{R}_{1}^{\times}$be the group of elements of $\mathcal{R}$ of 
reduced norm 1 , and let $\Gamma_{\infty}:=\iota_{\infty}\left(\mathcal{R}_{1}^{\times}\right) \subset \mathbf{S L}_{2}(\mathbb{R})$. Then the Riemann surface $X(\mathbb{C})$ can be described as the quotient $\mathcal{H} / \Gamma_{\infty}$. See [BD 5] for more details.

Assume that $S-\{\infty\}$ is non-empty and let $p \in S$ be a rational prime. Let $B$ be the (definite) quaternion algebra ramified precisely at the places in $S-\{p\}$, and let $R$ be an Eichler $\mathbb{Z}[1 / p]$-order in $B$ of level $N^{+}$. Let us fix an isomorphism $\iota: B \otimes \mathbb{Q}_{p} \longrightarrow M_{2}\left(\mathbb{Q}_{p}\right)$. It induces a group homomorphism, also denoted $\iota$ by abuse of notation, $\iota: B^{\times} \longrightarrow \mathbf{P G L}_{2}\left(\mathbb{Q}_{p}\right)$. Let $\Gamma=\iota\left(R_{1}^{\times}\right) \subset$ $\mathbf{P S L}_{2}\left(\mathbb{Q}_{p}\right)$ be the image under $\iota$ of the elements of norm 1 in $R$. Then the rigid analytic curve $X\left(\mathbb{C}_{p}\right)$ can be described as the quotient $\mathcal{H}_{p} / \Gamma$.

This description of the Shimura curve $X$ follows from a theorem of CerednikDrinfeld, see $[\mathrm{Ce}]$ and $[\mathrm{Dr}]$. Detailed proofs of these results can be found in $[\mathrm{JL} 1]$ and $[\mathrm{BC}]$.

$p$-adic modular forms. For the following definition recall the quotient $X\left(\mathbb{C}_{p}\right)=\mathcal{H}_{p} / \Gamma$ from the paragraph on Shimura curves above. For all $M=$ $\left(\begin{array}{ll}a & b \\ c & d\end{array}\right) \in \mathbf{G L}_{2}\left(\mathbb{C}_{p}\right)$, write

$$
\left(\left.f\right|_{k} M\right)(z):=\frac{\operatorname{det}(M)^{k / 2}}{(c z+d)^{k}} f(M z) .
$$

Definition 1.1 A p-adic modular form of weight $k$ on $\Gamma$ (or on $X$ ) is a global rigid analytic function $f$ on $\mathcal{H}_{p}$ satisfying

$$
\left.f(\gamma z)=(c z+d)^{k} f(z), \text { (i.e., }\left.f\right|_{k} \gamma=f\right), \quad \text { for all } \gamma=\left(\begin{array}{cc}
a & b \\
c & d
\end{array}\right) \in \Gamma \text {. }
$$

We denote by $S_{k}^{r i g}(\Gamma)$ the space of $p$-adic modular forms of weight $k$ for the group $\Gamma$.

Hecke operators. Let $N$ be a positive integer, and fix a prime $p$ which divides $N$ exactly. The space $S_{k}\left(\Gamma_{0}(N)\right)$ of cusp forms of weight $k$ on $\Gamma_{0}(N)$, and the space $S_{k}^{\text {new }}\left(\Gamma_{0}(N)\right)$ of newforms on this group, are endowed with an action of the commuting Hecke operators $T_{n}$ for each $n \geq 1$, defined in the standard way. (See for example $[\mathrm{MTT}]$, ch. I, §4.) Note that, following the conventions of [MTT], when $\ell$ is a prime dividing $N$ the symbol $T_{\ell}$ is used to denote the Hecke operator sometimes written $U_{\ell}$ in other articles. 
The space $S_{k}\left(\Gamma_{0}(N)\right)$ is also acted on by the Atkin-Lehner involutions $W_{\ell}$ for each prime $\ell \mid N$. (In [MTT], ch. I, $\S 5$, the involution $W_{\ell}$ is called $w_{\ell}$, where $\ell^{a}$ is the maximal power of $\ell$ dividing $N$.) The normalized newforms in $S_{k}\left(\Gamma_{0}(N)\right)$ are also eigenvectors for these involutions. Let $w= \pm 1$ denote the negative of the eigenvalue of $W_{p}$ acting on $\phi$,

$$
W_{p}(\phi)=-w \phi, \quad \text { so that } \quad T_{p}(\phi)=w p^{\frac{k-2}{2}} \phi .
$$

The form $\phi$ is said to be of split multiplicative type if $w=1$, and of non-split multiplicative type if $w=-1$.

Let $S$ be a set of places of $\mathbb{Q}$ of odd cardinality containing $\{\infty\}$, and suppose that

$$
N=N^{+} \prod_{\ell \in S-\infty} \ell
$$

with $N^{+}$not divisible by any prime in $S$. Let $X$ be the Shimura curve attached to the data $\left(S, N^{+}\right)$. By abuse of notation, let $T_{n}$ denote the $n$-th Hecke correspondence on $X$, defined for example as in [JL2]. When $\ell \notin S$ is a prime which does not divide $N^{+}$, (resp. divides $N^{+}$), the correspondence $T_{\ell}$ is of bidegree $\ell+1$ (resp. $\ell$ ), just like its $X_{0}(N)$-counterpart. When $\ell$ belongs to $S$, the operator $T_{\ell}$ arises from an an involution on $X$. (Cf. for example [BD1], sec. 1.5, where $T_{p}$ is denoted $W_{p}^{-}$.)

The Jacquet-Langlands correspondence. Crucial to the constructions of this paper is the Jacquet-Langlands correspondence which allows the normalised newform $\phi=\sum a_{n} q^{n}$ on $\Gamma_{0}(N)$ to be replaced by a modular form on a Shimura curve associated to the appropriate quaternion algebra. Let $X$ be the Shimura curve defined in the previous section.

Theorem 1.2 (Jacquet-Langlands) There exists a p-adic modular form $f$ of weight $k$ for the group $\Gamma$ satisfying

$$
T_{\ell}(f)=a_{\ell} f, \quad \forall \ell \notin S .
$$

This function is unique, up to scaling by a non-zero scalar in $\mathbb{C}_{p}$.

The group $\Gamma$ arising in the $p$-adic uniformisation of $X$ is contained in $\tilde{\Gamma}:=$ $\iota\left(R^{\times}\right) \subset \mathbf{P G L}_{2}\left(\mathbb{Q}_{p}\right)$ with index two. Choose any element $\tilde{\gamma} \in \tilde{\Gamma}-\Gamma$. Then

$$
\left.f\right|_{k} \tilde{\gamma}=w f
$$


where the reader is reminded that $w$ is the negative of the sign of the AtkinLehner involution $W_{p}$ acting on $\phi$. Thus $f$ is $\tilde{\Gamma}$-invariant if and only if $\phi$ is of split multiplicative type at $p$.

\subsection{Harmonic cocycles}

Harmonic cocycles are treated in both [Sch] and [Tei] but as our point of view is slightly different we prefer to restate the definitions and main properties of these objects here. Let $\Gamma$ be the subgroup of $\mathbf{P S L}_{2}\left(\mathbb{Q}_{p}\right)$ defined above and let $M$ be a $\mathbb{C}_{p}[\Gamma]$-module, where the action of $\Gamma$ is written on the left.

Definition 1.3 An $M$-valued harmonic cocycle on $\mathcal{T}$ is an $M$-valued function on $\overrightarrow{\mathcal{E}}(\mathcal{T})$ satisfying

$$
c(e)=-c(\bar{e}), \quad \sum_{\operatorname{source}(e)=v} c(e)=0, \quad \forall v \in \mathcal{T} .
$$

Write $C_{\text {har }}(M)$ for the $\mathbb{C}_{p}$-vector space of $M$-valued harmonic cocycles, and $C_{\text {har }}(M)^{\Gamma}$ for the space of $\Gamma$-equivariant harmonic cocycles, i.e., harmonic cocycles $c$ satisfying

$$
c(\gamma e)=\gamma \cdot c(e), \quad \text { for all } \gamma \in \Gamma .
$$

Let $\mathcal{P}_{k-2}$ be the $(k-1)$-dimensional $\mathbb{C}_{p}$-vector space of polynomials of degree $\leq k-2$ with coefficients in $\mathbb{C}_{p}$. It is endowed with a right action of $\mathbf{G L}_{2}\left(\mathbb{Q}_{p}\right)$ (and actually of $\mathbf{P G L}_{2}\left(\mathbb{Q}_{p}\right)$ ) by the rule

$$
P(x) \cdot \beta:=\frac{(c x+d)^{k-2}}{(\operatorname{det}(\beta))^{\frac{k-2}{2}}} P\left(\frac{a x+b}{c x+d}\right), \quad \beta=\left(\begin{array}{cc}
a & b \\
c & d
\end{array}\right), \quad P \in \mathcal{P}_{k-2} .
$$

In this way $\mathcal{P}_{k-2}^{\vee}:=\operatorname{hom}\left(\mathcal{P}_{k-2}, \mathbb{C}_{p}\right)$ inherits a left $\mathbf{P G L}_{2}\left(\mathbb{Q}_{p}\right)$-action by

$$
\beta \cdot R(P):=R(P \cdot \beta), \quad \forall P \in \mathcal{P}_{k-2} \text {. }
$$

Let $U:=\operatorname{ad}^{0}(B)$ be the representation of $B^{\times}$consisting of the elements of $B$ of reduced trace zero on which $B^{\times}$acts on the right by the rule

$$
u \cdot b:=\left(b^{-1} u b\right)
$$


The function which to every $u \in U$ associates the polynomial $P_{u}(x) \in \mathcal{P}_{2}$ (with coefficients in $\mathbb{Q}_{p}$ ) given by

$$
P_{u}(x)=\operatorname{trace}\left(\iota(u)\left(\begin{array}{cc}
x & -x^{2} \\
1 & -x
\end{array}\right)\right)=\operatorname{trace}\left(\iota(u)\left(\begin{array}{l}
x \\
1
\end{array}\right)\left(\begin{array}{ll}
1 & -x
\end{array}\right)\right)
$$

satisfies $P_{u \cdot b}(x)=P_{u}(x) \cdot \iota(b)$, and hence induces a linear map from $U \otimes \mathbb{C}_{p}$ to $\mathcal{P}_{2}$ which intertwines the $B^{\times}$and $\mathbf{P G L}\left(\mathbb{Q}_{p}\right)$ actions.

The symmetric bilinear $\mathbb{Q}$-valued pairing on $U$ arising from the CartanKilling form on $\operatorname{ad}^{0}(B)$,

$$
\left\langle u_{1}, u_{2}\right\rangle_{1}:=\frac{1}{2} \operatorname{trace}\left(u_{1} \bar{u}_{2}\right)
$$

satisfies $\left\langle u_{1} \cdot b, u_{2}\right\rangle_{1}=\left\langle u_{1}, u_{2} \cdot \bar{b}\right\rangle_{1}$, so that $\left\langle u_{1} \cdot b, u_{2} \cdot b\right\rangle_{1}=\left\langle u_{1}, u_{2}\right\rangle_{1}$. This pairing gives rise to a perfect symmetric pairing $\langle,\rangle_{j}$ on $\operatorname{Sym}^{j}(U)$ by the rule

$$
\left\langle u_{1} \cdots u_{j}, v_{1} \cdots v_{j}\right\rangle_{j}=\frac{1}{j !} \sum_{\sigma \in S_{j}}\left\langle u_{1}, v_{\sigma 1}\right\rangle_{1} \cdots\left\langle u_{j}, v_{\sigma j}\right\rangle_{1} .
$$

Thus the space $\operatorname{Sym}^{\frac{k-2}{2}}(U)$ is identified with its own dual via the pairing $\langle,\rangle_{\frac{k-2}{2}}$. Dualizing the natural surjection

$$
\operatorname{Sym}^{\frac{k-2}{2}}(U) \otimes \mathbb{C}_{p} \longrightarrow \mathcal{P}_{k-2}
$$

induced from the map $U \longrightarrow \mathcal{P}_{2}$ of equation (3) yields an inclusion

$$
\mathcal{P}_{k-2}^{\vee} \longrightarrow \operatorname{Sym}^{\frac{k-2}{2}}(U) \otimes \mathbb{C}_{p}
$$

In this way $\mathcal{P}_{k-2}^{\vee}$ inherits a perfect bilinear pairing arising from $\langle,\rangle_{\frac{k-2}{2}}$, denoted simply by $\langle$,$\rangle .$

If $L$ is any subfield of $\mathbb{C}_{p}$, write $\mathcal{P}_{k-2}(L)$ for the natural image of the map $\operatorname{Sym}^{\frac{k-2}{2}}(U) \otimes L \longrightarrow \mathcal{P}_{k-2}$ arising from equation (5), and let $\mathcal{P}_{k-2}^{\vee}(L):=$ $\operatorname{hom}\left(\mathcal{P}_{k-2}(L), L\right)$. This definition yields a rational structure on $\mathcal{P}_{k-2}$ and $\mathcal{P}_{k-2}^{\vee}$. Note that in general $\mathcal{P}_{k-2}(\mathbb{Q})$ is not the space of polynomials with rational coefficients. 
Definition 1.4 $A$ harmonic cocycle of weight $k$ on $\mathcal{T}$ is a $\mathcal{P}_{k-2}^{\vee}$-valued harmonic cocycle.

Set $C_{\text {har }}(k):=C_{\text {har }}\left(\mathcal{P}_{k-2}^{\vee}\right)$, and $C_{\text {har }}(k)^{\Gamma}:=C_{\text {har }}\left(\mathcal{P}_{k-2}^{\vee}\right)^{\Gamma}$.

Following Schneider [Sch], [Tei], associate to a rigid analytic modular form $f$ of weight $k$ on $\Gamma$ a harmonic cocycle $c_{f} \in C_{h a r}(k)$ by the rule

$$
c_{f}(e)(r)=\operatorname{res}_{e}(f(z) r(z) d z), \quad r(z) \in \mathcal{P}_{k-2},
$$

where res $_{e}$ is the $p$-adic annular residue along the oriented wide open annulus $V(e)$ in $\mathbb{P}_{1}\left(\mathbb{C}_{p}\right)$, defined by

$$
\operatorname{res}_{e}(\omega):=\operatorname{res}_{V(e)}\left(\left.\omega\right|_{V(e)}\right) .
$$

The fact that $c_{f}$ is harmonic follows from the $p$-adic residue formula. (Cf. [Sch].)

Definition 1.5 For $\gamma \in \mathbf{P G L}_{2}\left(\mathbb{Q}_{p}\right)$ denote by $|\gamma|:=\operatorname{ord}_{p}(\operatorname{det}(\bar{\gamma}))(\bmod 2)$, where $\bar{\gamma}$ is any lift of $\gamma$ in $\mathbf{G L}_{2}\left(\mathbb{Q}_{p}\right)$.

Lemma 1.6 The cocycle $c_{f}$ satisfies the following transformation with respect to $\tilde{\Gamma}$

$$
c_{f}(\gamma e)=w^{|\gamma|} \gamma \cdot c_{f}(e), \quad \forall \gamma \in \tilde{\Gamma} .
$$

Proof: For all $r \in \mathcal{P}_{k-2}$, and $\gamma=\left(\begin{array}{cc}a & b \\ c & d\end{array}\right)$,

$$
\begin{aligned}
c_{f}(\gamma e)(r) & =\operatorname{res}_{\gamma e}(f(z) r(z) d z)=\operatorname{res}_{e}(f(\gamma z) r(\gamma z) d(\gamma z)) \\
& =\operatorname{res}_{e}\left(w^{|\gamma|} \frac{(c z+d)^{k}}{(\operatorname{det} \gamma)^{\frac{k}{2}}} f(z) \frac{(c z+d)^{2-k}}{(\operatorname{det} \gamma)^{\frac{2-k}{2}}}(r(z) \cdot \gamma) \frac{\operatorname{det} \gamma}{(c z+d)^{2}} d z\right) \\
& =w^{|\gamma|} \operatorname{res}_{e}(f(z)(r(z) \cdot \gamma) d z)=w^{|\gamma|} c_{f}(e)(r(z) \cdot \gamma) \\
& =w^{|\gamma|} \gamma \cdot c_{f}(e)(r) .
\end{aligned}
$$

Define a pairing on $C_{h a r}(k)^{\Gamma}$ by the rule

$$
\left\langle c_{1}, c_{2}\right\rangle=\sum_{e \in \overrightarrow{\mathcal{E}}(\mathcal{T}) / \Gamma} w_{e}\left\langle c_{1}(e), c_{2}(e)\right\rangle,
$$


where the sum is taken over a set of representatives for the $\Gamma$-orbits in $\overrightarrow{\mathcal{E}}(\mathcal{T})$, the integer $w_{e}$ is the cardinality of the stabiliser of $e$ in $\Gamma$, and the pairing on $\mathcal{P}_{k-2}^{\vee}$ is the one defined by equation (6) and the sentence after it. The pairing on $C_{h a r}(k)^{\Gamma}$ is non-degenerate, because of the non-degeneracy of the pairing on $\mathcal{P}_{k-2}^{\vee}$ used to define it. It can be checked directly that the Hecke operators $T_{\ell}$ for $\ell \chi N$ are self-adjoint with respect to $\langle$,$\rangle .$

Let now $\phi$ be a normalized newform of weight $k \geq 2$ on $\Gamma_{0}(N)$ and $f \in S_{k}^{\text {rig }}(\Gamma)$ be the rigid analytic modular form on $\Gamma$ attached to it by section 1.1. The associated cocycle $c_{f} \in C_{\text {har }}(k)^{\Gamma}$ belongs to a one-dimensional simultaneous eigenspace for the Hecke algebra, by theorem 1.2. Hence the non-degeneracy of the pairing $\langle$,$\rangle on C_{h a r}(k)^{\Gamma}$ implies that

$$
\left\langle c_{f}, c_{f}\right\rangle \neq 0 \text {. }
$$

Note that the form $f$ is only well-defined up to multiplication by a non-zero scalar; the following definition is introduced to remove this ambiguity.

Definition 1.7 An eigenform $f \in S_{k}^{r i g}(\Gamma)$ is said to be normalised if its associated cocycle $c_{f} \in C_{\text {har }}(k)$ satisfies

$$
\left\langle c_{f}, c_{f}\right\rangle=1
$$

Note that the normalised eigenform $f \in S_{k}^{r i g}(\Gamma)$ attached to $\phi$ is well defined, up to a sign. Suppose from now on that $f$ is normalised in this way.

Recall that $K_{\phi} \subset \mathbb{C}_{p}$ is the finite extension of $\mathbb{Q}$ generated by the Fourier coefficients of $\phi$. The normalised eigenform $f$ satisfies the following rationality property.

Lemma 1.8 The $\mathcal{P}_{k-2}^{\vee}$-valued cocycle $c_{f}$ takes values in $\mathcal{P}_{k-2}^{\vee}\left(K_{f}\right)$, where $K_{f}$ is an extension of $K_{\phi}$ of degree $\leq 2$.

Proof: The space of $\Gamma$-equivariant $\mathcal{P}_{k-2}^{\vee}(\mathbb{Q})$-valued cocycles gives a rational structure $C_{h a r}(k)_{\mathbb{Q}}^{\Gamma}$ on $C_{h a r}(k)^{\Gamma}$ which is preserved by the Hecke operators, and on which the pairing $\langle$,$\rangle takes values in \mathbb{Q}$. Hence the one-dimensional eigenspace of $C_{\text {har }}(k)^{\Gamma}$ attached to $\phi$ contains a $K_{\phi}$-rational vector $\tilde{c}_{f} \in$ $C_{\text {har }}(k)_{\mathbb{Q}}^{\Gamma} \otimes K_{\phi}$. Since $\left\langle\tilde{c}_{f}, \tilde{c}_{f}\right\rangle$ belongs to $K_{\phi}$, the lemma follows, with $K_{f}=$ $K_{\phi}\left(\sqrt{\left\langle\tilde{c}_{f}, \tilde{c}_{f}\right\rangle}\right)$. 


\subsection{Schneider's distribution}

This section reviews a construction of Schneider which associates to a rigid analytic modular form $f$ of weight $k$ on $\Gamma$ a "boundary distribution" $\mu_{f}$.

An end of $\mathcal{T}$ is an equivalence class of sequences $\left(e_{n}\right)_{n=1}^{\infty}$ of elements $e_{n} \in$ $\overrightarrow{\mathcal{E}}(\mathcal{T})$ satisfying $\operatorname{target}\left(e_{n}\right)=\operatorname{source}\left(e_{n+1}\right)$, and $\operatorname{target}\left(e_{n+1}\right) \neq \operatorname{source}\left(e_{n}\right)$, two such sequences $\left(e_{n}\right)$ and $\left(e_{n}^{\prime}\right)$ being identified if there exist $N$ and $N^{\prime}$ with $e_{N+j}=e_{N^{\prime}+j}^{\prime}$ for all $j \geq 0$. Let $\mathcal{E}_{\infty}(\mathcal{T})$ be the space of ends on $\mathcal{T}$. It is identified with $\mathbb{P}_{1}\left(\mathbb{Q}_{p}\right)$ by the rule

$$
\left(e_{n}\right) \mapsto \lim _{n} b_{e_{n}}(\infty),
$$

where $b_{e_{n}}$ is the coset in $\mathbf{P G L}_{2}\left(\mathbb{Q}_{p}\right)$ associated to $e_{n}$. (See [BD 5] §3.1.) The space $\mathcal{E}_{\infty}(\mathcal{T})$ thus inherits a natural topology coming from the $p$-adic topology on $\mathbb{P}_{1}\left(\mathbb{Q}_{p}\right)$. Each edge $e \in \overrightarrow{\mathcal{E}}(\mathcal{T})$ corresponds to a compact open subset $U(e)$ of $\mathcal{E}_{\infty}(\mathcal{T})$ consisting of all ends having a representative which contains $e$.

Locally analytic distributions. We would like now to recall a few facts on $p$-adic distributions. We will adopt the point of view and notations from [St].

Suppose that $X \subset \mathbb{P}_{1}\left(\mathbb{C}_{p}\right)$ is a compact subset. Let us recall the following well known definition. Let $\alpha, \beta \in \mathbb{P}_{1}\left(\mathbb{C}_{p}\right)$ be represented by $\alpha=\left(a_{1}, a_{2}\right)$ and $\beta=\left(b_{1}, b_{2}\right)$ with $a_{1}, a_{2}, b_{1}, b_{2} \in \mathcal{O}_{\mathbb{C}_{p}}$ such that g.c.d. $\left(a_{1}, a_{2}\right)=$ g.c.d. $\left(b_{1}, b_{2}\right)=1$ and set $d(\alpha, \beta):=p^{-\operatorname{ord}_{p}\left(a_{1} b_{2}-a_{2} b_{1}\right)}$. Then $d$ is a well defined non-archimedean metric on $\mathbb{P}_{1}\left(\mathbb{C}_{p}\right)$. For every integer $n \geq 1$ denote by

$$
X[n]:=\left\{x \in \mathbb{P}_{1}\left(\mathbb{C}_{p}\right) \quad \mid \quad \text { there is } \quad y \in X \quad \text { with } \quad d(x, y) \leq p^{-n}\right\} .
$$

Then $X[n]$ is an affinoid subdomain of $\mathbb{P}_{1}\left(\mathbb{C}_{p}\right)$, in fact it is a finite disjoint union of affinoid balls. Let $A(X[n])$ be the $\mathbb{Q}_{p}$-algebra of rigid analytic functions on $X[n]$, and denote by $\rho^{(n)}: A(X[n]) \longrightarrow A(X[n+1])$ the restriction map. Then for each $n \geq 1, A(X[n])$ is a Banach algebra over $\mathbb{Q}_{p}$ for the spectral norm $\|\bullet\|_{n}$ and $\rho^{(n)}$ is a continuous homomorphism of Banach algebras. Let us denote

$$
A(X):=\lim _{\rightarrow} A(X[n])
$$

and call it the $\mathbb{Q}_{p}$-algebra of locally analytic functions on $X$. We endow this algebra with the Morita topology. 
Let us now fix $X=\mathbb{P}_{1}\left(\mathbb{Q}_{p}\right)$ and write simply $\mathcal{A}$ for $A(X)$. Let us go back to the notations at the beginning of this section. The cocycle $c_{f}$ associated to $f$ by equation (7) gives rise to a continuous linear functional $\mu_{f}$ on the space of locally analytic functions on $\mathbb{Q}_{p}$ with compact support, defined by the basic relation

$$
\mu_{f}\left(r \cdot \chi_{U(e)}\right):=\int_{U(e)} r(x) d \mu_{f}(x)=c_{f}(e)(r),
$$

for all $r \in \mathcal{P}_{k-2}$ and $e$ edge of $\mathcal{T}$ such that $U(e) \subset \mathbb{Q}_{p}$. (See $\left[\right.$ Sch].) Here $\chi_{U(e)}$ denotes the characteristic function of $U(e)$ in $\mathbb{Q}_{p}$. Then $\mu_{f}$ extends uniquely to the space, denoted $\mathcal{A}_{k}$, of locally analytic $\mathbb{C}_{p}$-valued functions on $\mathbb{P}_{1}\left(\mathbb{Q}_{p}\right)$ having a pole of order at most $k-2$ at $\infty$. (See [Tei], proposition 9.) This linear functional will be also denoted by $\mu_{f}$.

Lemma 1.9 If $r$ is any polynomial of degree $\leq k-2$, then

$$
\int_{\mathbb{P}_{1}\left(\mathbb{Q}_{p}\right)} r(x) d \mu_{f}(x)=0 .
$$

Proof: Let $v$ be any vertex of $\mathcal{T}$. By the finite additivity of $\mu_{f}$,

$$
\int_{\mathbb{P}_{1}\left(\mathbb{Q}_{p}\right)} r(x) d \mu_{f}(x)=\sum_{e, \operatorname{source}(e)=v} \int_{U(e)} r(x) d \mu_{f}(x) .
$$

The lemma follows from (8) combined with the harmonicity of $c_{f}$.

Now define a weight $k-2$ action of $\mathbf{P G L}_{2}\left(\mathbb{Q}_{p}\right)$ on $\mathcal{A}_{k}$ by the rule

$$
(\varphi * \gamma)(x):=\frac{(c x+d)^{k-2}}{(\operatorname{det} \gamma)^{\frac{k-2}{2}}} \varphi(\gamma * x) \quad \text { for } \quad \varphi \in \mathcal{A}_{k}, \quad \gamma \in \mathbf{P G L}_{2}\left(\mathbb{Q}_{p}\right)
$$

We have

Lemma 1.10 The boundary distribution $\mu_{f}$ satisfies the following transformation property with respect to $\tilde{\Gamma}$

$$
\mu_{f}(\varphi * \gamma)=w^{|\gamma|} \mu_{f}(\varphi) \quad \text { for all } \quad \gamma \in \tilde{\Gamma} \quad \text { and } \quad \varphi \in \mathcal{A} .
$$


Proof: It is enough to check the formula for functions of the form $r \cdot \chi_{U(e)}$, where $r$ is any polynomial of degree $\leq k-2, e$ any edge of $\mathcal{T}$ and now $\chi_{U(e)}$ is the characteristic function of $U(e)$ in $\mathbb{P}_{1}\left(\mathbb{Q}_{p}\right)$. For this apply lemma 1.6 and the definition of $\mu_{f}$.

Remark: Let $P \in \mathcal{P}_{k-2}$ having coefficients in $\mathbb{Q}_{p}$. Let us consider the free $\mathcal{A}$-module of rank one $M_{P}:=P \cdot \mathcal{A} \subset \mathcal{A}_{k}$ and endow it with the topology induced from the Morita topology of $\mathcal{A}$. Then the restriction of $\mu_{f}$ to $M_{P}$ is a continuous linear functional i.e. a distribution. (See [Tei] proposition 9.)

The $p$-adic Poisson transform. The following result can be viewed as a $p$-adic analogue of the Poisson inversion formula: it allows a rigid analytic modular form to be recovered from its associated boundary distribution.

Proposition 1.11 (Teitelbaum) Let $f$ be a rigid analytic modular form of weight $k$ on $\Gamma$ and let $\mu_{f}$ be the associated distribution on $\mathbb{P}_{1}\left(\mathbb{Q}_{p}\right)$. Then

$$
f(z)=\int_{\mathbb{P}_{1}\left(\mathbb{Q}_{p}\right)} \frac{1}{z-t} d \mu_{f}(t) .
$$

Proof: See [Tei], theorem 3. Note that the integrand $\frac{1}{z-t}$ is a bounded analytic function of $t$ so that the integral in the theorem converges.

\section{The $p$-adic $L$-function}

Let $K$ be an imaginary quadratic field. The goal of this chapter is to define the anticyclotomic $p$-adic $L$-function attached to $\phi$ and $K$. As explained in section 2.2, its construction is inspired by Schneider's "rigid analytic" definition [Sch] of $p$-adic $L$-functions in the cyclotomic case. For simplicity, the following assumption is made throughout.

Assumption 2.1 The discriminant of $K$ is relatively prime to the level $N$ of $\phi$.

Thus one may write

$$
N=p N^{+} N^{-},
$$

where $N^{+}$(resp. $N^{-}$) is divisible only by primes which are split (resp. inert) in $K$. The following assumption is also made for simplicity:

Assumption 2.2 The integer $N^{-}$is square-free. 


\subsection{Complex $L$-functions}

The classical $L$-function attached to $\phi$ is defined by

$$
L(\phi, s)=\prod_{\ell \mid N}\left(1-a_{\ell} \ell^{-s}\right)^{-1} \prod_{\ell \mid N}\left(1-a_{\ell} \ell^{-s}+\ell^{k-1-2 s}\right)^{-1}=\sum_{n=1}^{\infty} a_{n} n^{-s},
$$

where the Euler product is taken over the rational primes. It has an analytic continuation and a functional equation relating its values at $s$ and $k-s$. More precisely, the function

$$
\Lambda(\phi, s):=N^{s / 2}(2 \pi)^{-s} \Gamma(s) L(\phi, s)
$$

extends holomorphically to the entire complex plane and satisfies

$$
\Lambda(\phi, s)=(-1)^{k / 2} w_{N} \Lambda(\phi, k-s),
$$

where $w_{N}= \pm 1$ is the eigenvalue of the Atkin-Lehner involution $W_{N}$ acting on $\phi$.

More germane to the present article is the $L$-function of $\phi$ over $K$, defined by

$$
L(\phi / K, s)=L(\phi, s) L(\phi, \varepsilon, s),
$$

where $\varepsilon$ is the quadratic Dirichlet character attached to $K$ and $L(\phi, \varepsilon, s)=$ $\sum a_{n} \epsilon(n) n^{-s}$ is the twisted $L$-function. For each prime $\ell \nmid N$ write $(x-$ $\left.\alpha_{\ell}\right)\left(x-\beta_{\ell}\right):=x^{2}-a_{\ell} x+\ell^{k-1}$, and set $a\left(\ell^{t}\right)=\alpha_{\ell}^{t}+\beta_{\ell}^{t}$. The function $L(\phi / K, s)$ factors into an Euler product,

$$
L(\phi / K, s)=\prod_{v \mid N}\left(1-a_{\mathbb{N} v} \mathbb{N} v^{-s}\right)^{-1} \prod_{v \downarrow N}\left(1-a(\mathbb{N} v) \mathbb{N} v^{-s}+\mathbb{N} v^{k-1-2 s}\right)^{-1},
$$

the product being taken this time over all the finite places $v$ of $K$. The function $L(\phi / K, s)$ can also be expressed as a sum of partial $L$-functions,

$$
L(\phi / K, s)=\sum_{\mathfrak{a}} L(\phi / K, \mathfrak{a}, s) .
$$

Here the a's range over the ideal classes of $K$, and

$$
L(\phi / K, \mathfrak{a}, s)=\left(\sum_{\substack{n=1 \\(n, N)=1}}^{\infty} \frac{\varepsilon(n)}{n^{2 s-k+1}}\right)\left(\sum_{n=1}^{\infty} \frac{a_{n} r_{\mathfrak{a}}(n)}{n^{s}}\right),
$$


where $r_{\mathfrak{a}}(n)$ is the number of integral ideals of norm $n$ in the class of $\mathfrak{a}$.

More generally, let $\mathcal{O}_{c}$ be the order of $K$ of conductor $c$, and let $G_{c}=$ $\operatorname{Pic}\left(\mathcal{O}_{c}\right)$ be the Picard group of rank one projective $\mathcal{O}_{c}$-modules. If $\mathfrak{a}$ is a class in $G_{c}$, define $L(\phi / K, \mathfrak{a}, s)$ as in equation (10). The group $G_{c}$ is identified with the Galois group of an abelian extension $K_{c}$ of $K$, the so-called ring class field of conductor $c$. If $\chi: G_{c} \longrightarrow \mathbb{C}^{\times}$is any character, set

$$
L(\phi / K, \chi, s):=\sum \chi(\mathfrak{a}) L(\phi / K, \mathfrak{a}, s),
$$

the sum being taken over all elements of $G_{c}$. Like $L(\phi / K, s)$, and unlike the partial $L$-functions $L(\phi / K, \mathfrak{a}, s)$, the functions $L(\phi / K, \chi, s)$ admit an Euler product decomposition analogous to (9), and a simple functional equation.

Proposition 2.3 Suppose that $\chi$ is a ring class character of conductor $p^{n}$ where $n>0$. The $L$-function $L(\phi / K, \chi, s)$ satisfies the functional equation

$\Lambda(\phi / K, \chi, s):=(2 \pi)^{-2 s} \Gamma(s)^{2}(N D)^{s} L(\phi / K, \chi, s)=-\varepsilon\left(N^{-}\right) \Lambda(\phi / K, \chi, k-s)$

relating its values at $s$ and $k-s$.

For a discussion in weight 2, see [PR], sec. 5.2.

The anticyclotomic $p$-adic $L$-function studied in this work interpolates the special values of $L(\phi / K, \chi, s)$ at the central point $s=k / 2$, where $\chi$ ranges over ring class characters of $p$-power conductor. Proposition 2.3 shows that $L(\phi / K, \chi, k / 2)$ vanishes identically for all such $\chi$, if the number of primes dividing $N^{-}$is even. This justifies the following basic assumption which is made throughout the paper:

Assumption 2.4 The sign $\epsilon_{K}:=-\epsilon\left(N^{-}\right)$in the functional equation of proposition 2.3 is equal to 1, i.e., in light of assumptions 2.1 and 2.2, $N^{-}$is a product of an odd number of primes.

\subsection{The basic strategy}

The construction of the $p$-adic $L$-function attached to $\phi$ and $K$ proceeds as follows. 
1. Write $N^{-}=\ell_{1} \cdots \ell_{r}$ for the prime factorisation of $N^{-}$, and set $S=$ $\left\{p, \infty, \ell_{1}, \ldots, \ell_{r}\right\}$. Note that $S$ is a set of places of odd cardinality, by assumption 2.4. Let $X$ be the Shimura curve associated to the data $\left(S, N^{+}\right)$as in section 1.1, and let $\omega$ be the modular form of weight $k$ on $X$ attached to $\phi$ by the Jacquet-Langlands theorem (theorem 1.2).

2. Applying the Cerednik-Drinfeld theorem, see 1.1 let $f \in S_{k}^{r i g}(\Gamma)$ be the rigid analytic modular form on $\mathcal{H}_{p}$ associated to $\omega$. It satisfies invariance properties under a $p$-adic discrete group $\Gamma$ arising from the definite quaternion algebra $B$ ramified at the primes in $S-\{p\}$. More precisely, $\Gamma=\iota\left(R_{1}^{\times}\right)$, where $R$ is an Eichler $\mathbb{Z}[1 / p]$-order of level $N^{+}$in $B$ and $R_{1}^{\times}$is the group of elements of reduced norm 1 in $R^{\times}$. Assume that $f$ is normalised as in definition 1.7.

3. The $p$-adic $L$-function $L_{p}(f, K, s)$ is defined as a $p$-adic Mellin transform of Schneider's distribution $\mu_{f}$ attached to $f$ as in section 1.3, along the $p$ adic points of a (suitably chosen) maximal torus in $B^{\times} / \mathbb{Q}^{\times}$, isomorphic to $K^{\times} / \mathbb{Q}^{\times}$.

It is the last step of this construction which remains to be explained. In sections 2.3 and 2.4, a distribution on $\left(K \otimes \mathbb{Q}_{p}\right)^{\times} / \mathbb{Q}_{p}^{\times}$is defined using Schneider's distribution. Section 2.5 explains how this distribution interpolates special values of classical $L$-functions of $\phi$ over $K$. The construction of the $p$-adic $L$-function is then carried out in section 2.6

\subsection{Embeddings of $K$ into $B$}

Let $\mathcal{O}_{K}$ be the ring of integers of $K$, and $\mathcal{O}=\mathcal{O}_{K}[1 / p]$ its ring of $p$-integers. An orientation of the Eichler order $R$ is a surjective ring homomorphism

$$
\mathfrak{o}: R \longrightarrow\left(\mathbb{Z} / N^{+} \mathbb{Z}\right) \times \prod_{\ell \mid N^{-}} \mathbb{F}_{\ell^{2}} .
$$

The pair $(R, \mathfrak{o})$ is called an oriented Eichler order. Likewise, an orientation on $\mathcal{O}$ is a surjective homomorphism $\mathcal{O} \longrightarrow\left(\mathbb{Z} / N^{+} \mathbb{Z}\right) \times \prod_{\ell \mid N^{-}} \mathbb{F}_{\ell^{2}}$. This amounts to choosing, for each prime $\ell$ dividing $N^{+}$, a prime ideal of $K$ above $\ell$, and, for each prime $\ell$ dividing $N^{-}$, an identification of the residue field of $K$ at $\ell$ with $\mathbb{F}_{\ell^{2}}$. Fix orientations on $R$ and $\mathcal{O}$ once and for all.

An embedding $\Psi: K \longrightarrow B$ is called an oriented optimal embedding of $\mathcal{O}$ into $R$ if 
1. $\Psi(K) \cap R=\Psi(\mathcal{O})$, so that $\Psi$ induces an embedding of $\mathcal{O}$ into $R$.

2. $\Psi$ is compatible with the chosen orientations on $\mathcal{O}$ and $R$ in the sense that the following diagram commutes

$$
\begin{array}{ccc}
\mathcal{O} & \stackrel{\Psi}{\longrightarrow} & R \\
\searrow & & \mathfrak{o} \swarrow \\
\left(\mathbb{Z} / N^{+}\right. & \mathbb{Z}) \times \prod_{\ell \mid N^{-}} \mathbb{F}_{\ell^{2}} .
\end{array}
$$

The group $R^{\times}$acts naturally by conjugation on the set of oriented optimal embeddings. Write $\operatorname{emb}(\mathcal{O}, R)$ for the set of all oriented optimal embeddings of $\mathcal{O}$ into $R$, taken modulo conjugation by $R_{1}^{\times}$.

Denote by $\Delta=\operatorname{Pic}(\mathcal{O})$ the Picard group of projective modules of rank one over $\mathcal{O}$. (In classical language, it is the quotient of the class group of $K$, by the subgroup generated by the classes of the prime ideals above $p$.) The group $\Delta$ acts naturally on $\operatorname{emb}(\mathcal{O}, R)$ as follows (see also [Gr2], sec. 1 and 3 ). Let $\mathfrak{a} \subset \mathcal{O}$ be a projective $\mathcal{O}$-module representing a class $\alpha \in \Delta$, chosen in such a way that $\mathfrak{a} \otimes \mathbb{Z}_{\ell}=\mathcal{O} \otimes \mathbb{Z}_{\ell}$ for all $\ell \mid N^{+} N^{-}$, and let $\Psi: K \longrightarrow B$ be an oriented optimal embedding of $\mathcal{O}$ into $R$. The right order of the left $R$-ideal $R \Psi(\mathfrak{a})$, denoted $R_{\mathfrak{a}}$, is an Eichler order of level $N^{+}$in $B$, which inherits an orientation from the one on $R$, since $R_{\mathfrak{a}} \otimes \mathbb{Z}_{\ell}=R_{\ell}$ for all $\ell \mid N^{+} N^{-}$. The right action of $\Psi(\mathcal{O})$ on $R \Psi(\mathfrak{a})$ yields an embedding $\tilde{\Psi}_{\mathfrak{a}}: \mathcal{O} \longrightarrow R_{\mathfrak{a}}$, which is compatible with the orientations on $\mathcal{O}$ and $R_{\mathfrak{a}}$. Since all Eichler $\mathbb{Z}[1 / p]$-orders of level $N^{+}$in $B$ are conjugate, there exists $a \in B^{\times}$such that

$$
\operatorname{ord}_{p}(a \bar{a}) \equiv 0 \quad(\bmod 2), \quad a \in R_{\ell}^{\times} \text {for all } \ell \mid N^{+} N^{-}, \quad R=a R_{\mathfrak{a}} a^{-1} .
$$

Set

$$
\alpha \cdot \Psi:=a \tilde{\Psi}_{\mathfrak{a}} a^{-1} .
$$

It can be checked that $\alpha \cdot \Psi$ is an oriented optimal embedding of $K$ into $B$, and that its image in $\operatorname{emb}(\mathcal{O}, R)$ depends only on the image of $\mathfrak{a}$ in $\operatorname{Pic}(\mathcal{O})$ and of $\Psi$ in $\operatorname{emb}(\mathcal{O}, R)$, so that (12) gives a well-defined action of $\Delta$ on $\operatorname{emb}(\mathcal{O}, R)$. The following result is proved as in [Gr2], sec. 3 .

Proposition 2.5 The group $\Delta=\operatorname{Pic}(\mathcal{O})$ acts freely on emb $(\mathcal{O}, R)$. The set $\operatorname{emb}(\mathcal{O}, R)$ is the disjoint union of two $\Delta$-orbits under this action.

In particular, $\operatorname{emb}(\mathcal{O}, R)$ is finite and has cardinality $2 h$, where $h=\# \Delta$. 


\subsection{The $p$-adic distribution attached to an embedding}

Let $\Psi: K \longrightarrow B$ be an oriented optimal embedding of $\mathcal{O}$ into $R$. It induces an embedding

$$
\Psi: K_{p} \longrightarrow B_{p}, \quad \text { where } \quad K_{p}:=K \otimes \mathbb{Q}_{p} .
$$

Let us recall that we have fixed an isomorphism $\iota: B_{p} \longrightarrow M_{2}\left(\mathbb{Q}_{p}\right)$. The map $\iota \Psi: K_{p} \longrightarrow M_{2}\left(\mathbb{Q}_{p}\right)$ induces an embedding of $K_{p}^{\times} / \mathbb{Q}_{p}^{\times}$into $\mathbf{P G L}_{2}\left(\mathbb{Q}_{p}\right)$, which will also be denoted by $\iota \Psi$ by abuse of notation. Thus $\Psi$ gives rise to an action of $K_{p}^{\times} / \mathbb{Q}_{p}^{\times}$on the boundary $\mathbb{P}_{1}\left(\mathbb{Q}_{p}\right)$ of $\mathcal{H}_{p}$ by the rule

$$
\alpha * x:=\iota \Psi(\alpha)(x), \quad \alpha \in K_{p}^{\times} / \mathbb{Q}_{p}^{\times}, \quad x \in \mathbb{P}_{1}\left(\mathbb{Q}_{p}\right) .
$$

Choose a base point $\star \in \mathbb{P}_{1}\left(\mathbb{Q}_{p}\right)$. The element $b_{\Psi}:=\Psi(\sqrt{-D})$ is an element of $B$ of reduced trace 0 . Let $P_{\Psi}$ be the polynomial $P_{b_{\Psi}}$ in $\mathcal{P}_{2}$ defined as in equation (3) of section 1.2. Note that

$$
P_{\Psi} \cdot(\iota \Psi(\alpha))=P_{\Psi},
$$

for all $\alpha \in K_{p}^{\times}$.

Case 1: $p$ is inert in $K$. The (compact) torus $\iota \Psi\left(K_{p}^{\times} / \mathbb{Q}_{p}^{\times}\right) \subset \mathbf{P G L}_{2}\left(\mathbb{Q}_{p}\right)$ acts simply transitively on the boundary $\mathbb{P}_{1}\left(\mathbb{Q}_{p}\right)$. The choice of $\star$ determines an isomorphism $\eta_{\Psi, \star}: K_{p}^{\times} / \mathbb{Q}_{p}^{\times} \longrightarrow \mathbb{P}_{1}\left(\mathbb{Q}_{p}\right)$. The torus $\iota \Psi\left(K_{p}^{\times}\right)$has two fixed points in $\mathcal{H}_{p}$, denoted $a$ and $\bar{a}$, which belong to $K_{p}$ and are interchanged by $\operatorname{Gal}\left(K_{p} / \mathbb{Q}_{p}\right)$. Let us now fix the base point $\star=\infty$. We have a natural homeomorphism $K_{p}^{\times} / \mathbb{Q}_{p}^{\times} \cong K_{p, 1}^{\times}$sending $x\left(\bmod \mathbb{Q}_{p}^{\times}\right)$to $x / \bar{x} \in K_{p, 1}^{\times}$. Here $K_{p, 1}^{\times}$denotes the compact subgroup of $K_{p}^{\times}$of elements of norm 1 . Let us denote by $\eta_{\Psi}$ the composition

$$
\eta_{\Psi}: G:=K_{p, 1}^{\times} \longrightarrow K_{p}^{\times} / \mathbb{Q}_{p}^{\times} \longrightarrow \mathbb{P}_{1}\left(\mathbb{Q}_{p}\right) .
$$

We then have the following formulas

$$
\eta_{\Psi}(\alpha)=\frac{(a \alpha-\bar{a})}{\alpha-1}, \quad \eta_{\Psi}^{-1}(x)=\frac{x-\bar{a}}{x-a} .
$$

Pullback by $\eta_{\Psi}$ and $\eta_{\Psi}^{-1}$ on functions preserve local analyticity and so we get a natural, continuous isomorphism

$$
\eta_{\Psi *}=\left(\eta_{\Psi}^{-1}\right)^{*}: A(G) \longrightarrow \mathcal{A},
$$


where the notations of section 1.3 are used. Recall that we have defined a natural, continuous linear functional $\mu_{f}$ on the free $\mathcal{A}$-module of rank one $P_{\Psi}^{\frac{k-2}{2}} \cdot \mathcal{A} \subset \mathcal{A}_{k}$ in section 1.3 . We will use it to define the locally analytic distribution $\mu_{f, \Psi, \star}$ on $G=K_{p, 1}^{\times}$, which will be simply denoted by $\mu_{\Psi}$ whenever $f$ and the basepoint $\star$ are fixed, by

$$
\mu_{f, \Psi, \star}(\varphi)=\mu_{\Psi}(\varphi):=\mu_{f}\left(P_{\Psi}^{\frac{k-2}{2}} \times\left(\eta_{\Psi}^{-1}\right)^{*}(\varphi)\right), \quad \text { for } \quad \varphi \in A(G) .
$$

Case 2: $p$ is split. The torus $\iota \Psi\left(K_{p}^{\times} / \mathbb{Q}_{p}^{\times}\right) \subset \mathbf{P G L}_{2}\left(\mathbb{Q}_{p}\right)$ has precisely two fixed points $a, b \in \mathbb{P}_{1}\left(\mathbb{Q}_{p}\right)$, and it acts simply transitively on the complement $\mathbb{P}_{1}\left(\mathbb{Q}_{p}\right)-\{a, b\}$. The choice of a base point $\star$ in this complement determines an identification

$$
\eta_{\Psi, \star}: K_{p}^{\times} / \mathbb{Q}_{p}^{\times} \longrightarrow \mathbb{P}_{1}\left(\mathbb{Q}_{p}\right)-\{a, b\}
$$

by the rule

$$
\eta_{\Psi, \star}(\alpha):=\alpha * \star .
$$

Choose $\star$ so that the Möbius transformation sending $(a, b, \star)$ to $(0,1, \infty)$ has the property that its determinant has even $p$-adic valuation. Base points satisfying this property will be called $\Psi$-normalised. Let $w(\alpha)$ be the locally constant function of $\alpha \in \mathbb{Q}_{p}^{\times}$defined by

$$
w(\alpha):=w^{o r d}(\alpha),
$$

where $w= \pm 1$ is the sign defined in section 1.1.

Now choose a prime $\mathfrak{p}$ of $K$ above $p$. This choice defines identifications $K_{p}^{\times} / \mathbb{Q}_{p}^{\times}=\left(K \otimes \mathbb{Q}_{p}\right)^{\times} / \mathbb{Q}_{p}^{\times}=\left(K_{\mathfrak{p}}^{\times} \times K_{\overline{\mathfrak{p}}}^{\times}\right) / \mathbb{Q}_{p}^{\times}$and a group isomorphism

$$
\epsilon: K_{p}^{\times} / \mathbb{Q}_{p}^{\times} \longrightarrow K_{\mathfrak{p}}^{\times}=\mathbb{Q}_{p}^{\times},
$$

which has the basic property that if $x \in K^{\times}$, then

$$
\epsilon([x])=\frac{x}{\bar{x}} \in K_{\mathfrak{p}}^{\times}=\mathbb{Q}_{p}^{\times} .
$$

Here $[x]$ denotes the image of $x$ in $K_{p}^{\times} / \mathbb{Q}_{p}^{\times}$. Let $u_{0}$ be a generator of the rank one group $\mathcal{O}^{\times} / \mathbb{Z}[1 / p]^{\times}$and let $u \in \mathbb{Q}_{p}^{\times}$be the image of $u_{0} / \bar{u}_{0}$ in $K_{\mathfrak{p}}=\mathbb{Q}_{p}$, 
normalised so that $\operatorname{ord}_{p}(u)>0$. In other words $u=\epsilon\left(\left[u_{0}\right]\right)$. Let us consider the group denerated by $u$, i.e. let $\sigma:=\langle u\rangle \subset \mathbb{Q}_{p}^{\times}$. Our next goal is to define a locally analytic distribution on $G:=\mathbb{Q}_{p}^{\times} / \sigma$.

Let us now fix a $\Psi$-normalized base point $\star \in \mathbb{P}_{1}\left(\mathbb{Q}_{p}\right)-\{a, b\}$, and denote by $\eta_{\Psi}$ the following composition

$$
\eta_{\Psi}: \mathbb{Q}_{p}^{\times} \stackrel{\epsilon^{-1}}{\longrightarrow} K_{p}^{\times} / \mathbb{Q}_{p}^{\times} \stackrel{\eta_{\Psi, \star}}{\longrightarrow} \mathbb{P}_{1}\left(\mathbb{Q}_{p}\right)-\{a, b\} .
$$

The map $\eta_{\Psi}$ satisfies the basic property that if $x, y \in \mathbb{Q}_{p}^{\times}$then

$$
\eta_{\Psi}(x y)=\Psi\left(\epsilon^{-1}(x)\right) * \eta_{\Psi}(y) .
$$

$\eta_{\Psi}$ extends to a fractional linear transformation $\eta_{\Psi}: \mathbb{P}_{1}\left(\mathbb{Q}_{p}\right) \longrightarrow \mathbb{P}_{1}\left(\mathbb{Q}_{p}\right)$ sending 0 to $a, \infty$ to $b$ and 1 to $\star$. Obviously, $\eta_{\Psi}$ extends uniquely to a fractional linear transformation denoted by the same symbol $\mathbb{P}_{1}\left(\mathbb{C}_{p}\right) \longrightarrow \mathbb{P}_{1}\left(\mathbb{C}_{p}\right)$.

In order to define the distribution $\mu_{f, \Pi, \star}$ when $p$ is split, we need to introduce locally analytic functions and distributions on $\mathbb{Q}_{p}^{\times}$with compact support. Let $X$ be a compact open subset of $\mathbb{Q}_{p}^{\times}$and $n$ an integer. Let us recall the $\mathbb{Q}_{p}$-topological algebras $A(X[n])$ and $A(X)$ from section 1.3. If $m, n$ are integers such that $m \geq n$ and $X, Y$ are compact open subsets of $\mathbb{Q}_{p}^{\times}$, with $X \subset Y$ then the restriction map $A(Y[n]) \longrightarrow A(X[m])$ is continuous and we denote

$$
\mathcal{A}_{c}:=\lim _{\rightarrow} A(X[n]),
$$

where the inductive limit is over pairs $(n, X)$ with $n$ integer and $X$ compact open subset of $\mathbb{Q}_{p}^{\times}$. Then $\mathcal{A}_{c}$ is the $\mathbb{Q}_{p}$-algebra of locally analytic functions on $\mathbb{Q}_{p}^{\times}$with compact support. We will endow it with the Morita topology. Let us now fix a parameter $z$ on $\mathbb{P}_{1}\left(\mathbb{Q}_{p}\right)$. This determines an embedding $\mathbb{Q}_{p}^{\times} \subset \mathbb{P}_{1}\left(\mathbb{Q}_{p}\right)$ and a continuous $\mathbb{Q}_{p}$-algebra homomorphism

$$
\psi_{!}: \mathcal{A}_{c} \longrightarrow \mathcal{A},
$$

which is extension by zero. Let us recall from section 1.3 that we have defined a natural, continuous linear functional $\mu_{f}$ on the $\mathcal{A}$-module $P_{\Psi}^{\frac{k-2}{2}} \cdot \mathcal{A} \subset \mathcal{A}_{k}$. We'll use $\mu_{f}$ to define a continuous linear functional $\mu_{f, \Psi, \star}$ on $\mathcal{A}_{c}$, which will be simply denoted by $\mu_{\Psi}$ whenever $f$ and $\star$ are fixed. Let $\varphi \in \mathcal{A}_{c}$ then

$$
\mu_{f, \Psi, \star}(\varphi)=\mu_{\Psi}(\varphi):=\mu_{f}\left(P_{\Psi}^{\frac{k-2}{2}}\left(\eta_{\Psi}^{-1}\right)^{*}\left(\psi_{!}(\varphi w)\right)\right)
$$


where $w$ is the function defined in equation (17). We will sometimes denote

$$
\mu_{f, \Psi, \star}(\varphi)=\int_{\mathbb{Q}_{p}^{\times}} \varphi d \mu_{f, \Psi, \star}
$$

We'll now use $\mu_{f, \Psi, \star}$ in order to define a locally analytic distribution on $G$. Let us denote by $\mathcal{F}$ a fundamental domain in $\mathbb{Q}_{p}^{\times}$for the action of $\sigma$, for example:

$$
\mathcal{F}:=\left\{x \in \mathbb{Q}_{p}^{\times} \quad|\quad p| u|\leq| x \mid \leq 1\right\} .
$$

Then $\mathcal{F}$ is a compact open subset of $\mathbb{Q}_{p}^{\times}$and $\mathbb{Q}_{p}^{\times}=\cup_{i \in \mathbb{Z}} u^{i} \mathcal{F}$. To give a function $\varphi: G \longrightarrow \mathbb{C}_{p}$ is equivalent to giving a function $\varphi: \mathbb{Q}_{p}^{\times} \longrightarrow \mathbb{C}_{p}$ such that $\varphi(u x)=\varphi(x)$ for all $x \in \mathbb{Q}_{p}^{\times}$. Such a function is determined by its restriction to $\mathcal{F}$. We say that a function $\varphi$ on $G$ is locally analytic if $\varphi \chi_{\mathcal{F}}$ is the restriction to $\mathbb{Q}_{p}^{\times}$of an element of $\mathcal{A}_{c}$, where $\chi_{\mathcal{F}}$ is the characteristic function of the set $\mathcal{F} \subset \mathbb{Q}_{p}^{\times}$.

Definition 2.6 Let $A(G)$ denote the $\mathbb{Q}_{p}$-algebra of locally analytic functions on $G$.

Let us remark that the map "restriction to $\mathcal{F}$ " defines an isomorphism between $A(\mathcal{F})$ and $A(G)$. We will identify these two algebras whenever convenient and endow $A(G)$ with the natural topology on $A(\mathcal{F})$.

We define an action of the discrete subgroup $\sigma \subset \mathbb{Q}_{p}^{\times}$on $\mathcal{A}_{c}$ by $(\varphi \times u)(x):=$ $\varphi(u x)$ for all $x \in \mathbb{Q}_{p}^{\times}$and $\varphi \in \mathcal{A}_{c}$. Obviously this action is continuous and it defines an action of $\sigma$ on linear functionals on $\mathcal{A}_{c}$.

Proposition $2.7 u \times \mu_{f, \Psi, \star}=\mu_{f, \Psi, \star}$, i.e. $\mu_{f, \Psi, \star}$ is $\sigma$-invariant.

Proof: First of all, we know that $\Psi\left(\epsilon^{-1}(u)\right)=\Psi\left(u_{0}\right) \in \tilde{\Gamma}$ and $w \times u=$ $w^{\text {ord }_{p}(u)} w$. Let $\varphi \in \mathcal{A}_{c}$ and let us write $\eta$ instead of $\eta_{\Psi}$ and $\mu_{\Psi}$ instead of $\mu_{f, \Psi, \star}$ for this proof. Then $\left(u \times \mu_{\Psi}\right)(\varphi)=\mu_{\Psi}(\varphi \times u)=\mu_{f}\left(P_{\Psi}^{\frac{k-2}{2}}\left(\eta^{-1}\right)^{*}\left(\psi_{!}((\varphi \times u) w)\right)\right)$.

Let us denote $g:=\psi_{!}(\varphi w)$. Then

$$
\begin{aligned}
& P_{\Psi}^{\frac{k-2}{2}}\left(\eta^{-1}\right)^{*}\left(\psi_{!}((\varphi \times u) w)\right)(x)=w^{\text {ord }_{p}(u)}\left(P_{\Psi}^{\frac{k-2}{2}}\left(\eta^{-1}\right)^{*}(g \times u)\right)(x) \\
= & w^{\text {ord }}(u) P_{\Psi}^{\frac{k-2}{2}}(x) g\left(u \eta^{-1}(x)\right)=w^{\text {ord }}(u) P_{\Psi}^{\frac{k-2}{2}}(x) g\left(\eta^{-1}\left(\Psi\left(u_{0}\right) * x\right)\right)
\end{aligned}
$$




$$
\begin{aligned}
& =w^{\operatorname{ord}_{p}(u)} P_{\Psi}^{\frac{k-2}{2}}(x)\left(\left(\eta^{-1}\right)^{*}(g)\right)\left(\Psi\left(u_{0}\right) * x\right) \\
& =w^{\operatorname{ord}_{p}(u)}\left[\left(P_{\Psi}^{\frac{k-2}{2}}\left(\eta^{-1}\right)^{*}(g)\right) * \Psi\left(u_{0}\right)\right](x) .
\end{aligned}
$$

Therefore $P_{\Psi}^{\frac{k-2}{2}}\left(\eta^{-1}\right)^{*}(g \times u)=w^{\operatorname{ord}_{p}(u)}\left(P_{\Psi}^{\frac{k-2}{2}}\left(\eta^{-1}\right)^{*}(g)\right) * \Psi\left(u_{0}\right)$. But ord $p(u)+$ $\left|\Psi\left(u_{0}\right)\right|=0(\bmod 2)$ and the result follows upon applying lemma 1.10.

Lemma 2.8 Let $V$ be a compact open subset of $\mathbb{Q}_{p}^{\times}$and $\varphi \in \mathcal{A}_{c}$. Then

$$
\int_{u^{i} V} \varphi d \mu_{\Psi}=\int_{V}\left(\varphi \times u^{i}\right) d \mu_{\Psi}
$$

for all $i \in \mathbb{Z}$.

Proof: We have

$$
\begin{gathered}
\int_{u^{i} V} \varphi d \mu_{\Psi}=\mu_{\Psi}\left(\varphi \chi_{u^{i} V}\right)=\mu_{\Psi}\left(\varphi\left(\chi_{V} \times u^{-i}\right)\right)= \\
=\mu_{\Psi}\left(\left(\left(\varphi \times u^{i}\right) \chi_{V}\right) \times u^{-i}\right)=\mu_{\Psi}\left(\left(\varphi \times u^{i}\right) \chi_{V}\right)=\int_{V}\left(\varphi \times u^{i}\right) d \mu_{\Psi} .
\end{gathered}
$$

Corollary 2.9 If $\varphi \in A(G)$ then

$$
\int_{u^{i} \mathcal{F}} \varphi d \mu_{\Psi}=\int_{\mathcal{F}} \varphi d \mu_{\Psi}
$$

for all $i \in \mathbb{Z}$.

Definition 2.10 The distribution $\mu_{f, \Psi, \star}$ on $\mathcal{A}_{c}$ induces a distribution, denoted also by $\mu_{f, \Psi, \star}$ (and by $\mu_{\Psi}$ whenever $f$ and $\star$ are fixed) on $G$ i.e. a continuous linear functional on $A(G)$ by

$$
\mu_{f, \Psi, \star}(\varphi)=\int_{G} \varphi d \mu_{f, \Psi, \star}:=\int_{\mathcal{F}} \varphi d \mu_{f, \Psi, \star}, \quad \text { for all } \varphi \in A(G) .
$$


For the rest of this section, the assumption that $w=1$ will be made. First of all

Remark: If $\varphi \in \mathcal{A}$ then $P_{\Psi}^{\frac{k-2}{2}}\left(\eta_{\Psi}^{-1}\right)^{*}(\varphi)$ is an element of the free $\mathcal{A}$-module of rank one $P_{\Psi}^{\frac{k-2}{2}} \cdot \mathcal{A}$. Therefore $\mu_{f, \Psi, \star}$ extends to a continuous linear functional on $\mathcal{A}$ denoted by the same symbol and given by the formula:

$$
\mu_{f, \Psi, \star}(\varphi)=\mu_{\Psi}(\varphi):=\mu_{f}\left(P_{\Psi}^{\frac{k-2}{2}}\left(\eta_{\Psi}^{-1}\right)^{*}(\varphi)\right) .
$$

We end this section with a few properties of the above defined distribution on $G$, which will be used in chapter 3 .

Lemma 2.11 Let $\varphi \in A\left[n_{0}\right]$ for some $n_{0} \in \mathbb{N}$ and suppose that $i_{0} \in \mathbb{N}$ is such that $\operatorname{ord}_{p}\left(u^{i_{0}}\right) \geq n_{0}$. Moreover suppose that $\varphi(0)=\varphi(\infty)=0$. Then there is a constant $C_{\varphi}$ such that for all $i \geq i_{0}$, if $V$ denotes the closed ball around 0 or around $\infty$ of radius $\left|u^{i}\right|$ then

$$
\left|\int_{V} \varphi d \mu_{\Psi}\right| \leq C_{\varphi}\left|u^{i}\right|
$$

Proof: It is enough to treat the case $V=B\left[0,\left|u^{i}\right|\right]$. Let $D:=B\left[0,\left|u^{i_{0}}\right|\right]$, then $V=u^{i-i_{0}} D$. Using the results above,

$$
\int_{u^{i-i_{0} D}} \varphi d \mu_{\Psi}=\int_{D}\left(\varphi \times u^{i-i_{0}}\right) d \mu_{\Psi}
$$

Because $\varphi(0)=0$, it follows that $\varphi(z)=\sum_{j \geq 1} a_{j} z^{j}$ for $z \in D$, and

$$
\left(\varphi \times u^{i-i_{0}}\right)(z)=u^{i-i_{0}}\left(\sum_{j \geq 1} a_{j} u^{(j-1)\left(i-i_{0}\right)} z^{j}\right) .
$$

Therefore

$$
\left|\int_{V} \varphi d \mu_{\Psi}\right| \leq\left|u^{i}\right|\left(\left|u^{-i_{0}}\right| \sup _{j \geq 1}\left(\left|a_{j} u^{(j-1)\left(i-i_{0}\right)} \int_{D} z^{j} d \mu_{\Psi}(z)\right|\right) \leq\left|u^{i}\right| C_{\varphi},\right.
$$

where

$$
C_{\varphi}:=\left|u^{-i_{0}}\right| \sup _{j \geq 1}\left|a_{j} \int_{D} z^{j} d \mu_{\Psi}(z)\right| .
$$

Note that the supremum is finite by the continuity of $\mu_{\Psi}$.

Finally we have the following 
Proposition 2.12 Let $\varphi \in \mathcal{A}$ be such that $\varphi(0)=\varphi(\infty)=0$. Then

a) $\sum_{i \in \mathbb{Z}}\left(\left.\varphi\right|_{\mathbb{Q}_{p}^{\times}} \times u^{i}\right) \in A(G)$.

b) We have

$$
\int_{\mathbb{P}_{1}\left(\mathbb{Q}_{p}\right)} \varphi d \mu_{\Psi}:=\mu_{\Psi}(\varphi)=\int_{G}\left(\left.\sum_{i \in \mathbb{Z}} \varphi\right|_{\mathbb{Q}_{p}^{\times}} \times u^{i}\right) d \mu_{\Psi} .
$$

Proof: a) Let us denote by $\psi(x):=\sum_{i \in \mathbb{Z}} \varphi\left(u^{i} x\right)$, for all $x \in \mathbb{Q}_{p}^{\times}$. Then the convergence of the series and the invariance of $\psi$ with respect to the action of $\sigma$ is clear. Moreover if denote by $\psi_{N}:=\sum_{i=-N}^{N}\left(\left.\varphi\right|_{\mathbb{Q}_{p}^{\times}} \times u^{i}\right)$ for every $N \in \mathbb{N}$, then one sees that $\psi=\lim _{N \rightarrow \infty} \psi_{N}$ where the limit is uniform on compact open subsets of $\mathbb{Q}_{p}^{\times}$. We leave it to reader to show that $\left.\psi\right|_{\mathcal{F}},\left.\psi_{N}\right|_{\mathcal{F}} \in A(G)$ for all $N$ and that the limit above is a limit in $A(G)$. In the application of this proposition to the proof of theorem 3.3 these facts will be obvious.

b) We have

$$
\begin{gathered}
\int_{\mathbb{P}_{1}\left(\mathbb{Q}_{p}\right)} \varphi d \mu_{\Psi}-\int_{\mathcal{F}} \psi_{N} d \mu_{\Psi}=\int_{\mathbb{P}\left(\mathbb{Q}_{p}\right)} \varphi d \mu_{\Psi}-\int_{\cup_{i=-N}^{N} u^{i} \mathcal{F}} \varphi d \mu_{\Psi}= \\
=\int_{B\left[0,\left|u^{N+1}\right|\right]} \varphi d \mu_{\Psi}+\int_{B\left[\infty,\left|u^{N}\right|\right]} \varphi d \mu_{\Psi} .
\end{gathered}
$$

For $N$ big enough, $\varphi \in A\left[\operatorname{ord}_{p}\left(u^{N}\right)\right]$ and from lemma 1.6 we have

$$
\left|\int_{\mathbb{P}\left(\mathbb{Q}_{p}\right)} \varphi d \mu_{\Psi}-\int_{\mathcal{F}} \psi_{N} d \mu_{\Psi}\right| \leq\left|u^{N}\right| C_{\varphi}^{\prime} \stackrel{N \rightarrow \infty}{\longrightarrow} 0
$$

The fact that $\mu_{\Psi}$ is a continuous linear functional on $\mathcal{A}_{c}$ imply that

$$
\lim _{N \rightarrow \infty} \int_{\mathcal{F}} \psi_{N} d \mu_{\Psi}=\int_{\mathcal{F}} \psi d \mu_{\Psi}=\int_{G} \psi d \mu_{\Psi}
$$

\subsection{Interpolation of classical special values}

This section describes how the distributions $\mu_{f, \Psi}$ interpolate special values of the classical $L$-function $L(\phi / K, s)$ at $s=k / 2$, justifying for the definition of the $p$-adic $L$-function that is given in section 2.6, definitions 2.19 and 2.20. The interpolation properties of $\mu_{f, \Psi}$ are summarized in formula 
(18) and proposition 2.18. Strictly speaking, these are not required for the construction of the $p$-adic $L$-function given in section 2.6 , but merely provide motivation and justification for it. Therefore, in a first reading, the reader may wish to skip directly to section 2.6 after looking at (18) and the statement of proposition 2.18, postponing the proofs for later.

Galois-theoretic preliminaries. An abelian extension $L / K$ is called anticyclotomic if it is Galois over $\mathbb{Q}$ and if the involution in $\operatorname{Gal}(K / \mathbb{Q})$ acts as -1 on $\operatorname{Gal}(L / K)$ by conjugation. Let $K_{\infty}$ denote the maximal anticyclotomic extension of $K$ which is unramified outside $p$. Let $H$ denote the Hilbert class field of $K$, and let $H_{p}$ be the field fixed by a Frobenius element at $p$ in $\operatorname{Gal}(H / K)$ (i.e., the maximal unramified abelian extension of $K$ in which $p$ splits completely). Let $K_{n}$ denote the ring class field of $K$ of conductor $p^{n}$, so that $K_{0}=H$. The fields $K_{n}$ form a tower of extensions containing the anticyclotomic $\mathbb{Z}_{p}$-extension:

$$
\mathbb{Q} \subset K \subset H_{p} \subset H \subset K_{1} \subset \cdots \subset K_{n} \subset \cdots .
$$

Class field theory provides a Galois theoretic interpretation of the $p$-adic group $G$ defined in section 2.4. More precisely,

$$
G=\operatorname{Gal}\left(K_{\infty} / H_{p}\right)
$$

if $\mathcal{O}_{K}^{\times}=\{ \pm 1\}$. For simplicity assume from now on that $\mathcal{O}_{K}^{\times}=\{ \pm 1\}$, a condition which is satisfied as soon as $\operatorname{Disc}\left(\mathcal{O}_{K}\right)<-4$. We denote

$$
G^{0}=\operatorname{Gal}\left(K_{\infty} / H\right), \quad G^{n}=\operatorname{Gal}\left(K_{\infty} / K_{n}\right), \quad \Delta=\operatorname{Gal}\left(H_{p} / K\right),
$$

(where $\Delta$ is the group defined in section 2.3). Write

$$
\tilde{G}:=\operatorname{Gal}\left(K_{\infty} / K\right) \text {. }
$$

This group fits into the exact sequence

$$
1 \longrightarrow G \longrightarrow \tilde{G} \longrightarrow \Delta \longrightarrow 1,
$$

and it can be identified with the Picard group of rank one projective $\mathcal{O}$ modules $\mathfrak{a}$ equipped with a trivialisation at $p$, i.e., a fixed isomorphism $K_{p} \longrightarrow \mathfrak{a} \otimes \mathbb{Q}_{p}$. More precisely, let $\hat{K}$ denotes the finite adèles of $K$, and write

$$
\hat{\mathcal{O}}=\prod_{\ell} \mathcal{O}_{\ell}, \quad \hat{\mathcal{O}}_{\#}=\prod_{\ell \neq p} \mathcal{O}_{\ell}
$$


Class field theory identifies $\tilde{G}$ with $\hat{K}^{\times} / K^{\times} \hat{\mathbb{Q}}^{\times} \hat{\mathcal{O}}_{\#}^{\times}$. An element in this coset space with representative $\left(g_{\ell}\right)$ corresponds to the projective module $\mathfrak{a}=$ $K \cap \prod_{\ell \neq p} \mathcal{O}_{\ell} g_{\ell}$, with trivialisation at $p$ sending 1 to $g_{p}$. Let $\Lambda$ be the set of pairs $(\Psi, \star)$ taken modulo conjugation by $R_{1}^{\times}$, where $\Psi$ is an oriented optimal embedding of $\mathcal{O}$ into $R$, and $c$ is a $\Psi$-normalised base point (if $p$ is inert in $K$, every base point is defined to be $\Psi$-normalised.)

Lemma 2.13 The action of $\Delta$ on $\operatorname{emb}(\mathcal{O}, R)$ lifts to a free action of $\tilde{G}$ on $\Lambda$, which is the union of two orbits under this action.

The description of this action proceeds along the same lines as in section 2.3. Let $\mathfrak{a} \subset \mathcal{O}$ be a representative for a class $\alpha \in \tilde{G}$, i.e., a projective rank one $\mathcal{O}$-module together with a $K_{p}$-generator $t$ for $\mathfrak{a} \otimes \mathbb{Q}_{p}$. Let $R_{\mathfrak{a}}$ denote the right order of the left $R$-ideal $R \Psi(\mathfrak{a})$ of section 2.3, and let $g=\Psi(t) \in R_{\mathfrak{a}} \otimes \mathbb{Q}_{p}$. Choosing an element $a \in B^{\times}$satisfying the conditions of equation (11) of section 2.3 , and letting $\tilde{\Psi}_{\mathfrak{a}}$ be the embedding defined there, set

$$
\alpha \cdot(\Psi, \star)=\left(\Psi^{\prime}, \star^{\prime}\right), \quad \text { where } \quad \Psi^{\prime}=a \tilde{\Psi}_{a} a^{-1}, \quad c^{\prime}=\iota\left(a g a^{-1}\right)(c) .
$$

By fixing a representative $\Psi$ for an element of $\operatorname{emb}(\mathcal{O}, R)$, and a $\Psi$-normalised base point $c$, a locally analytic distribution $\mu_{f, K}$ on $\tilde{G}$ will be defined as follows. First, for all $\delta \in \Delta$ choose and fix a lift $\alpha_{\delta} \in \tilde{G}$. This allows us to view $\tilde{G}$ as the disjoint union of the orbits $\alpha_{\delta} G$ for $\delta \in \Delta$. Let $\varphi: \tilde{G} \longrightarrow \mathbb{C}_{p}$ be a function. We say that $\varphi$ is locally analytic if

$$
\left(\left.\varphi\right|_{\alpha_{\delta} G}\right) \times \alpha_{\delta} \in A(G) \text { for all } \delta \in \Delta,
$$

where let us recall $\left(\varphi \times \alpha_{\delta}\right)(x):=\varphi\left(\alpha_{\delta} x\right)$ for all $x \in G$. The set of locally analytic functions on $\tilde{G}$ will be denoted $A(\tilde{G})$. To define $\mu_{f, K}$, let $\varphi \in A(\tilde{G})$. Then set

$$
\mu_{f, K}(\varphi)=\int_{\tilde{G}} \varphi d \mu_{f, K}:=\sum_{\delta \in \Delta} \mu_{f, \Psi_{\delta}, \star_{\delta}}\left(\left.\varphi\right|_{\alpha_{\delta} G} \times \alpha_{\delta}\right),
$$

where, if $\delta \in \Delta$ then $\left(\Psi_{\delta}, \star_{\delta}\right)=\alpha_{\delta}(\Psi, \star)$.

For further applications, we want to calculate the values of $\mu_{f, K}$ on locally constant functions on $\tilde{G}$.

Lemma 2.14 For all $\alpha \in \tilde{G}$,

$$
\mu_{f, K}\left(\alpha G^{n}\right)=\mu_{f, \Psi^{\prime}, \star^{\prime}}\left(G^{n}\right), \quad \text { where }\left(\Psi^{\prime}, \star^{\prime}\right)=\alpha \cdot(\Psi, \star) .
$$


Proof: Let $\delta$ be the projection of $\alpha$ on $\Delta$. Then, by the definition above,

$$
\mu_{f, K}\left(\alpha G^{n}\right):=\mu_{f, K}\left(\chi_{\alpha G^{n}}\right)=\mu_{f, \Psi_{\delta},{ }_{\delta}}\left(a G^{n}\right):=\mu_{f, \Psi_{\delta}, \star_{\delta}}\left(\chi_{a G^{n}}\right),
$$

where $a=\alpha_{\delta}^{-1} \alpha \in G$. We have

$$
\left(\Psi^{\prime}, \star^{\prime}\right)=\alpha(\Psi, \star)=a\left(\alpha_{\delta}(\Psi, \star)\right)=a\left(\Psi_{\delta}, \star_{\delta}\right)=\left(\Psi_{\delta}, \Psi_{\delta}(a) * c_{\delta}\right) .
$$

A calculation, using the fact that both $c^{\prime}$ and $c_{\delta}$ are $\Psi^{\prime}=\Psi_{\delta}$ normalised based points, shows that

$$
\mu_{f, \Psi_{\delta},{ }_{\delta}}\left(a G^{n}\right)=\mu_{f, \Psi_{\delta}, \Psi_{\delta}(a) *{ }_{\delta}}\left(G^{n}\right)=\mu_{f, \Psi^{\prime}, \star^{\prime}}\left(G^{n}\right) .
$$

The lemma shows that the restriction of $\mu_{f, K}$ to locally constant functions is independent of the choices of the $\alpha_{\delta}$ 's. Moreover

Lemma 2.15 The distribution $\mu_{f, K}$ depends on the choice of $(\Psi, \star)$, only up to translation by an element of $\tilde{G}$, and up to multiplication by $w$. Its restriction to $G$ is equal to $\mu_{f, \Psi, \star}$.

Proof: If $(\Psi, \star)$ is replaced by $\left(\gamma \Psi \gamma^{-1}, \gamma \star\right)$ with $\gamma \in R_{1}^{\times}$, the associated distribution is unchanged, by lemma 1.10. If $(\Psi, \star)$ and $\left(\Psi^{\prime}, \star^{\prime}\right)=\alpha \cdot(\Psi, \star$ are in the same $\tilde{G}$-orbit, the associated distributions differ by translation by $\alpha$. Finally, if $(\Psi, \star)$ and $\left(\Psi^{\prime}, \star^{\prime}\right)$ belong to different $\tilde{G}$-orbits, the associated distributions differ by translation by an element of $\tilde{G}$, and multiplication by $w$.

Choose a complex embedding of the field $K_{f}$ introduced in lemma 1.8. Since the cocycle $c_{f}$ takes values in $\mathcal{P}_{k-2}^{\vee}\left(K_{f}\right)$ (lemma 1.8), the $p$-adic distribution $\mu_{f, K}$ can also be viewed as a complex-valued distribution, against which locally constant $\mathbb{C}$-valued functions on $\tilde{G}$ can be integrated.

In particular, let $\chi: \tilde{G} \longrightarrow \mathbb{C}^{\times}$be a continuous character of finite order. By lemma 2.15, the integral $\int_{\tilde{G}} \chi(\alpha) d \mu_{f, K}(\alpha)$ depends on the choice of $(\Psi, \star)$ only up to multiplication by a root of unity, so that its complex norm is well defined.

Define the multiplier $M(\chi)$ by

$$
M(\chi)=\left\{\begin{array}{l}
1 \text { if } \chi \text { is ramified; } \\
\left(1-w \chi\left(\text { frob }_{\mathfrak{p}}\right)\right)\left(1-w \bar{\chi}\left(\text { frob }_{\mathfrak{p}}\right)\right) \text { otherwise }
\end{array}\right.
$$


Write $u_{K}=\# \mathcal{O}_{K}^{\times} / 2$, let $D_{K}$ be the discriminant of $K$, and denote by $(\phi, \phi)$ the Petersson scalar product of $\phi$ with itself.

The distribution $\mu_{f, K}$ is expected to satisfy the following interpolation property with respect to special values of the $L$-function of $\phi$ over $K$.

$$
\left|\int_{\tilde{G}} \chi(x) d \mu_{f, K}(x)\right|^{2}=M(\chi) u_{K}^{2} D_{K}^{\frac{k-1}{2}} \frac{L(\phi / K, \chi, k / 2)}{(\phi, \phi)} .
$$

The remainder of this section elucidates the relation between (18) and the calculations explained in [Gr2] for weight $k=2$ and in [Ha] for general even weight. These articles treat the case - too restrictive for the application to (18) - of a modular form $\phi$ of prime level $N$, and an unramified character $\chi$. Specializing temporarily to the setting considered by [Gr2] and [Ha], take $N^{-}=N$ to be prime and set $N^{+}=1$. Let $B$ denote the definite quaternion algebra ramified at $N^{-}$and choose a maximal $\mathbb{Z}$-order $\underline{R}$ of $B$. Let $B_{0}$ be the space of elements of $B$ of trace 0 , and set

$$
\underline{\hat{R}}:=\prod_{\ell}\left(\underline{R} \otimes \mathbb{Z}_{\ell}\right), \quad \hat{B}:=\underline{\hat{R}} \otimes \mathbb{Q} .
$$

The article $[\mathrm{Ha}]$ introduces a vector bundle $V$ by the rule

$$
V=\left(\underline{\hat{R}}^{\times} \backslash \hat{B}^{\times} \times \operatorname{Sym}^{\frac{k-2}{2}}\left(B_{0}\right)\right) / B^{\times},
$$

where the action of $B^{\times}$on $\operatorname{Sym}^{\frac{k-2}{2}}\left(B_{0}\right)$ is as described in page 543 of [Ha]. Thanks to equation (3) of section 1.2, the Picard group of $V$ maps to the space of $\mathcal{P}_{k-2}$-valued functions on $\underline{\hat{R}}^{\times} \backslash \hat{B}^{\times}$which are invariant under the action of $B^{\times}$. Let $P(V)$ denote this image, i.e.,

$$
P(V):=\left\{c: \underline{\hat{R}}^{\times} \backslash \hat{B}^{\times} \longrightarrow \mathcal{P}_{k-2} \mid c(x b)=c(x) \cdot \iota(b) \text {, for all } b \in B^{\times}\right\} .
$$

It is a finite-dimensional $\mathbb{C}_{p}$-vector space since an element $c$ is entirely determined by its values on a system of representatives for the finite double coset space $\underline{\hat{R}}^{\times} \backslash \hat{B}^{\times} / B^{\times}$(cf. [Vi], ch. V). The space $P(V)$ is also endowed with a linear action of the Hecke operators $T_{n}$ defined as in [Ha], sec. 4 , and is isomorphic, as a Hecke module, to the space of modular forms of weight $k$ on $\Gamma_{0}\left(N^{-}\right)$with $\mathbb{C}_{p^{-} \text {-coefficients. }}$

In the paragraph before ch. 4 of [Ha], the author defines Heegner elements in $V$. These elements are described by pairs $\left(\Psi, \underline{R}_{\Psi}\right)$ modulo conjugation by $B^{\times}$, where 
1. $\underline{R}_{\Psi}$ is a maximal $\mathbb{Z}$-order of $B$. (By strong approximation there are only finitely many such orders, up to conjugation in $B^{\times}$.)

2. $\Psi: K \longrightarrow B$ is an oriented embedding which is optimal relative to $\mathcal{O}_{K}$ and $\underline{R}_{\Psi}$, in the sense that $\Psi(K) \cap \underline{R}_{\Psi}=\Psi\left(\mathcal{O}_{K}\right)$.

Such a pair yields an element in $V$ represented by $\left(\left(b_{\ell}\right)_{\ell} ; \Psi(\sqrt{-D})^{\frac{k-2}{2}}\right)$, where $\left(b_{\ell}\right)$ represents the coset describing $\underline{R}_{\Psi}$ as in [Ha]. The group $\operatorname{Pic}\left(\mathcal{O}_{K}\right)$ acts transitively on the set of Heegner elements in a manner similar to proposition 2.5. (Cf. also $[\mathrm{Ha}]$.)

Fix a Heegner element $\left(\Psi, \underline{R}_{\Psi}\right)$, and, for $\mathfrak{a} \in \operatorname{Pic}\left(\mathcal{O}_{K}\right)$ let

$$
v_{\mathfrak{a}}=\mathfrak{a} \cdot\left(\Psi, \underline{R}_{\Psi}\right),
$$

viewed as an element of $P(V)$. If $\chi$ is any character of $\operatorname{Pic}\left(\mathcal{O}_{K}\right)$, write $v_{\chi}=\sum_{\mathfrak{a}} \chi(\mathfrak{a}) v_{\mathfrak{a}}$, and let $v_{f, \chi} \in P(V) \otimes \mathbb{C}$ be the projection of this vector to the $f$-isotypic component for the action of the Hecke algebra. Recall the natural pairing $\langle$,$\rangle of equation (6) of section 1.2. It naturally induces a$ symmetric pairing on the spaces $C_{h a r}(k)$ and $C_{h a r}\left(\mathcal{P}_{k-2}\right)^{\Gamma}$ of $\Gamma$-equivariant harmonic cocycles, as well as on the space $P(V)$. Use the same symbol $\langle$, to denote these pairings, by abuse of notation. The following formula is proved by Gross in weight 2 [Gr2], and by Hatcher for even weight $k \geq 2$ (cf. proposition 8.2. of [Ha]):

\section{Proposition 2.16 (Gross, Hatcher)}

$$
\left\langle v_{f, \chi}, v_{f, \bar{\chi}}\right\rangle=u_{K}^{2} D_{K}^{\frac{k-1}{2}} \frac{L(\phi / K, \chi, k / 2)}{(\phi, \phi)} .
$$

Remark: Note that the factor $\left(\frac{k-2}{2}\right) !^{2}$ which is present in prop. 8.2 of [Ha] does not appear in proposition 2.16, because of the different normalisation that is used for the inner product on $\mathcal{P}_{k-2}^{\vee}$. (Compare equation (4) of section 1.2 with equation $(3.1)$ of $[\mathrm{Ha}]$.

To establish equation (18), it is first necessary to capture the modular forms on $\Gamma_{0}\left(N^{+} N^{-} p\right)$. In order to do this, introduce an auxiliary $\Gamma_{0}\left(N^{+} p\right)$ structure and replace the maximal order $\underline{R}$ by an Eichler order of level $N^{+} p$ in the definite quaternion algebra of discriminant $N^{-}$, to be denoted by the same letter, $\underline{R}$, from now on. By strong approximation, the double coset 
space $\underline{\hat{R}}^{\times} \backslash \hat{B}^{\times} / B^{\times}$is identified with $\underline{R}_{p}^{\times} \backslash B_{p}^{\times} / R^{\times}$, where $R:=\underline{R}[1 / p]$. Note that $R$ is an Eichler $\mathbb{Z}[1 / p]$-order of level $N^{+}$in $B$, as in the previous sections. Under $\iota$ the space $\underline{R}_{p}^{\times} \backslash B_{p}^{\times}$is identified with the space $\overrightarrow{\mathcal{E}}(\mathcal{T})$ of edges of $\mathcal{T}$. Thus $P(V)$ is identified with the space of functions $\tilde{c}: \overrightarrow{\mathcal{E}}(\mathcal{T}) \longrightarrow \mathcal{P}_{k-2}$ satisfying

$$
\tilde{c}\left(\gamma^{-1} e\right)=\tilde{c}(e) \cdot \gamma, \quad \text { for all } \gamma \in \tilde{\Gamma}=\iota\left(R^{\times}\right) .
$$

Suppose that $\tilde{c}$ is an eigenfunction for the Hecke correspondences satisfying $T_{n} \tilde{c}=a_{n} \tilde{c}$ for all $n$. The fact that $\phi$ is new at $p$ implies that

$$
\sum_{\operatorname{source}(e)=v} \tilde{c}(e)=0, \quad \sum_{\operatorname{target}(e)=v} \tilde{c}(e)=0, \quad \text { for all } v \in \mathcal{V}(\mathcal{T}) .
$$

(Cf. [BD3], prop. 1.4.) However, $\tilde{c}$ does not satisfy the rule $\tilde{c}(\bar{e})=-\tilde{c}(e)$ in general, but merely the formula

$$
\tilde{c}(\bar{e})=-w \tilde{c}(e),
$$

where $-w$ is the eigenvalue of the Atkin-Lehner involution at $p$ acting on $\phi$, which is equal to -1 when $\phi$ is of split multiplicative type, and 1 otherwise.

To turn $\tilde{c}$ into a harmonic cocycle when $\phi$ is of non-split multiplicative type, recall the base vertex $v^{\circ}$ on $\mathcal{T}$ and call a vertex even (resp. odd) if its distance from $v^{\circ}$ is even (resp. odd). An edge is then said to be positively oriented if its source is even and its target is odd, and negatively oriented otherwise. Note that this orientation is reversed by elements in $\tilde{\Gamma}-\Gamma$, but is preserved by the index two subgroup $\Gamma$. Now given $\tilde{c}$, define similarly as in the proof of prop. 1.4 of [BD3],

$$
c(e)=\left\{\begin{array}{l}
\tilde{c}(e) \text { if } e \text { is positively oriented } \\
-\tilde{c}(\bar{e}) \text { if } e \text { is negatively oriented }
\end{array}\right.
$$

The assignment $\tilde{c} \mapsto c$ defines a Hecke equivariant isomorphism between the space of $p$-new vectors in $P(V)$ and the space $C_{h a r}\left(\mathcal{P}_{k-2}\right)^{\Gamma}$ of $\Gamma$-equivariant harmonic cocycles on $\mathcal{T}$ with values in $\mathcal{P}_{k-2}$.

For the purposes of equation (18), it is also necessary to generalize the notion of Heegner elements. A Heegner element of level $p^{n}$ is now described by a pair $\left(\Psi, \underline{R}_{\Psi}\right)$, taken modulo conjugation by $B^{\times}$, satisfying 
1. $\underline{R}_{\Psi}$ is an Eichler order of level $N^{+} p$ in $B$; by strong approximation it can be assumed without loss of generality that $\underline{R}_{\Psi}[1 / p]=R$, and this is done from now on.

2. $\Psi$ is an optimal embedding of $\mathcal{O}_{n}$, the order in $\mathcal{O}_{K}$ of conductor $p^{n}$, i.e., $\Psi(K) \cap \underline{R}_{\Psi}=\Psi\left(\mathcal{O}_{n}\right)$. Note that when $n \geq 1$, such optimal embeddings always exist, whereas an optimal embedding of $\mathcal{O}_{K}$ into an Eichler order of level $N^{+} p$ only exists if $p$ is split in $K / \mathbb{Q}$.

To give a pair $\left(\Psi, \underline{R}_{\Psi}\right)$ as above is equivalent to giving a pair $(\Psi, e)$ satisfying

1. $\Psi(K) \cap R=\Psi(\mathcal{O})$,

2. $e$ is an edge of $\mathcal{T}$ which is at distance $n$ from the vertices fixed by $\iota \Psi\left(G^{0}\right)$.

For example, the Eichler order $\underline{R}_{\Psi}$ is equal to $R \cap \iota^{-1}\left(M_{e}\right)$, where $M_{e} \subset$ $M_{2}\left(\mathbb{Z}_{p}\right)$ is the local Eichler order of level $p$ attached to the edge $e$. The group $\tilde{G}_{n}=\operatorname{Pic}\left(\mathcal{O}_{n}\right)$ acts naturally on the set of Heegner elements of level $p^{n}$, and the action of $G=K_{p}^{\times} / \mathbb{Q}_{p}^{\times}$is given by

$$
\alpha \cdot(\Psi, e)=(\Psi, \iota \Psi(\alpha) e) .
$$

Fix a Heegner element $\left(\Psi, \underline{R}_{\Psi}\right)$ of level $p^{n}$ and write as before $v_{\mathfrak{a}}:=\mathfrak{a} \cdot\left(\Psi, \underline{R}_{\Psi}\right)$, with $\mathfrak{a} \in \tilde{G}_{n}$. Since it is only the images of the elements $v_{\mathfrak{a}}$ in $P(V)$ which matter in the calculations, the $v_{\mathfrak{a}}$ are viewed as elements of $P(V)$ from now on. The projection of $v_{\mathfrak{a}}$ onto the $f$-isotypic component of $C_{h a r}\left(\mathcal{P}_{k-2}\right)^{\Gamma}$, denoted $v_{\mathfrak{a}, f}$, can be viewed as elements of $C_{\text {har }}\left(\mathcal{P}_{k-2}\right)^{\Gamma}$ by the identification of the previous paragraph, and can be written as

$$
v_{\mathfrak{a}, f}=\left\langle v_{\mathfrak{a}}, c_{f}\right\rangle c_{f}^{\vee},
$$

where $c_{f}^{\vee}$ is the vector attached to $f$ in the basis for $C_{\text {har }}\left(\mathcal{P}_{k-2}\right)^{\Gamma}$ dual to the basis of normalised eigenforms in $C_{h a r}(k)^{\Gamma}$. It follows from the definition of $\mu_{f, K}$ that, after a suitable choice of base point $(\Psi, \star)$ of $\Lambda$, compatible with the choice $\left(\Psi, \underline{R}_{\Psi}\right)$ made to define $v_{\mathfrak{a}}$, that

$$
\left\langle v_{\mathfrak{a}, f}, c_{f}\right\rangle=\mu_{f, K}\left(\tilde{\mathfrak{a}} \cdot G^{n}\right),
$$

where $\tilde{\mathfrak{a}}$ is any lift of $\mathfrak{a} \in \tilde{G}_{n}$ to $\tilde{G}$. Hence if $\chi$ factors through $\tilde{G}_{n}$,

$$
\left\langle v_{f, \chi}, c_{f}\right\rangle= \pm \int_{\tilde{G}} \chi(x) d \mu_{f, K}(x), \text { where } v_{f, \chi}=\sum \chi(\mathfrak{a}) v_{\mathfrak{a}, f} \text {. }
$$

The following generalization of the proposition 2.16 is expected to hold 
Conjecture 2.17 : In the notations above,

$$
\begin{aligned}
& \left\langle v_{f, \chi}, v_{f, \bar{\chi}}\right\rangle=M(\chi) u_{K}^{2} D_{K}^{\frac{k-1}{2}} \frac{L(\phi / K, \chi, k / 2)}{(\phi, \phi)}, \text { if } n=0 ; \\
& \left\langle v_{f, \chi}, v_{f, \bar{\chi}}\right\rangle=u_{K}^{2} D_{K}^{\frac{k-1}{2}} \frac{L(\phi / K, \chi, k / 2)}{(\phi, \phi)} \text { if } n>0 .
\end{aligned}
$$

Remark The identities of conjecture 2.17 should follow from a direct generalization of the calculations carried on in [Gr2] and [Ha], but these calculations are not present in the literature. See also [Dag] and [Va] for a discussion of related topics.

Granting conjecture 2.17 and combining it with formula (19) yields

$$
\left|\int_{\tilde{G}} \chi(x) d \mu_{f, K}(x)\right|^{2}=M(\chi) u_{K}^{2} D_{K}^{\frac{k-1}{2}} \frac{L(\phi / K, \chi, k / 2)}{(\phi, \phi)},
$$

and (18) follows.

Suppose now that $p$ is split and that $\Psi_{j}(1 \leq j \leq h)$ is one of the oriented optimal embeddings of $\mathcal{O}$ into $R$. Choose an even vertex $v_{j}$ of $\mathcal{T}$ whose stabiliser under the action of $K_{p}^{\times}$induced by $\Psi_{j}$ is $\left(\mathcal{O}_{K} \otimes \mathbb{Z}_{p}\right)^{\times}$. Let us now recall the notations of section 1.3 i.e. $u_{0}$ be a generator of the rank one group $\mathcal{O}^{\times} / \mathbb{Z}[1 / p]^{\times}$such that the image $u$ of $\frac{u_{0}}{\bar{u}_{0}}$ in $\mathbb{Q}_{p}^{\times}$has $\operatorname{ord}_{p}(u)>0$. Denote by $\delta_{j}=\iota \Psi_{j}\left(u_{0}\right)$. Given an ordered edge $e$, let $\operatorname{sgn}(e)$ be equal to 0 if $e$ is positively oriented, and 1 if it is negatively oriented.

Proposition 2.18 Assume conjecture 2.17. Then

$$
\left(\sum_{j=1}^{h} \sum_{v_{j} \rightarrow \delta_{j} v_{j}} w^{s g n(e)} c_{f}(e)\left(P_{\Psi_{j}}^{\frac{k-2}{2}}\right)\right)^{2}=u_{K}^{2} D_{K}^{\frac{k-1}{2}} \frac{L(\phi / K, k / 2)}{(\phi, \phi)}
$$

where the inner sum on the left is taken over all edges in the path joining $v_{j}$ to $\delta_{j} v_{j}$.

Proof: We will apply formula (18) to the trivial character $\chi_{\text {triv }}$. Let us fix a $j$ as above and calculate

$$
\int_{G} \chi_{t r i v} d \mu_{f, \Psi_{j}, \star_{j}}=\int_{\mathcal{F}} w(\alpha) d \mu_{f, \Psi_{j}, \star_{j}}(\alpha)
$$


where $c_{j}$ is the corresponding $\Psi_{j}$-normalised base point, and let us recall from section 1.3 that $\mathcal{F}$ is a fundamental domain for the action of $u$ on $\mathbb{Q}_{p}^{\times}$. Let now $E_{j}$ denote the set of oriented edges of $\mathcal{T}$ with same source as the edges on the path from $v_{j}$ to $\delta_{j} v_{j}$, but not containing these edges. The geometry of the action of $\delta_{j}$ on $\mathcal{T}$, as explained in [BD4] $\S 5$, implies that the set

$$
\mathcal{F}^{\prime}:=\cup_{e \in E_{j}} U(e)
$$

is a fundamental domain for the action of $\delta_{j}$ on $\mathbb{P}_{1}\left(\mathbb{Q}_{p}\right)-\left\{a_{j}, b_{j}\right\}$. Therefore, $\eta_{\Psi_{j}}^{-1}\left(\mathcal{F}^{\prime}\right)$ is a fundamental domain for the action of $u$ on $\mathbb{Q}_{p}^{\times}$. Using the fact that the base point $c_{j}$ is $\Psi_{j}$-normalised we have

$$
\begin{gathered}
\int_{\mathcal{F}} w(\alpha) d \mu_{f, \Psi_{j}, \star_{j}}(\alpha)=\int_{\mathcal{F}^{\prime}} w(\alpha) P_{\Psi_{j}}^{\frac{k-2}{2}} d \mu_{f}(\alpha)= \\
=\sum_{e \in E_{j}} \int_{U(e)} w(\alpha) P_{\Psi_{j}}^{\frac{k-2}{2}} d \mu_{f}(\alpha)=\sum_{e \in E_{j}} w^{s g n(e)} c_{f}(e)\left(P_{\Psi_{j}}^{\frac{k-2}{2}}\right) .
\end{gathered}
$$

Proposition 2.18 now follows using the harmonicity of $c_{f}$ and the definition of $E_{j}$.

Remark: The path on $\mathcal{T}$ joining $v_{j}$ to $\delta_{j} v_{j}$ is a $p$-adic analogue of the geodesic paths on the classical upper half plane associated by Shintani to narrow ideal classes in real quadratic fields. The integrals of classical modular forms against such paths yield special values of their $L$-series over the associated real quadratic field. For this reason, the path in $\mathcal{H}_{p}$ from $z_{0}$ to $\delta_{j} * z_{0}$, or the path in $\mathcal{T}$ from $v_{j}$ to $\delta_{j} v_{j}$, are sometimes called $p$-adic Shintani cycles attached to the optimal embeddings $\Psi_{j}(1 \leq j \leq h)$. See for example [BD4].

\subsection{The $p$-adic $L$-function}

If $p$ is split in $K$, let log : $\mathbb{C}_{p}^{\times} \longrightarrow \mathbb{C}_{p}$ be a branch of the $p$-adic logarithm, normalised so that $\log (u)=0$. If $p$ is inert, let log be the usual branch satisfying $\log (p)=0$ (although any other choice would do equally well). The logarithm gives a homomorphism $\log : K_{\mathfrak{p}}^{\times} \longrightarrow K_{\mathfrak{p}}$ which is 0 on $\mathcal{O}_{1}^{\times}$, and hence, by passing to the quotient, a homomorphism from $G$ to $K_{\mathfrak{p}}$ which extends uniquely to a homomorphism

$$
\log : \tilde{G} \longrightarrow K_{\mathfrak{p}} \subset \mathbb{C}_{p} .
$$


For $\alpha \in \tilde{G}$ and $s \in \mathbb{Z}_{p}$, define

$$
\alpha^{s}:=\exp (s \log (\alpha)),
$$

where exp is the usual $p$-adic exponential. Note that $s \mapsto \alpha^{s}$ is an analytic function of $s \in \mathbb{Z}_{p}$.

Definition 2.19 The p-adic $L$-function attached to the distribution $\mu_{f, K}$ is the function of the $p$-adic variable $s \in \mathbb{Z}_{p}$ defined by

$$
L_{p}(f, K, s)=\int_{\tilde{G}} \alpha^{s-k / 2} d \mu_{f, K}(\alpha) .
$$

Definition 2.20 Let $\Psi$ be a representative for a class in emb $(\mathcal{O}, R)$ and let $c$ be a base point. The partial $p$-adic $L$-function attached to the datum $(\Psi, \star)$ is the function of the $p$-adic variable $s \in \mathbb{Z}_{p}$ defined by

$$
L_{p}(f, \Psi, \star, s)=\int_{G} \alpha^{s-k / 2} d \mu_{f, \Psi, \star}(\alpha) .
$$

Remark: If $c^{\prime}=\iota \Psi\left(\alpha_{0}\right) c$, then

$$
L_{p}\left(f, \Psi, \star^{\prime}, s\right)=\left(\alpha_{0}\right)^{s-\frac{k}{2}} L_{p}(f, \Psi, \star, s) .
$$

In particular,

Lemma 2.21 The order of vanishing of $L_{p}(f, \Psi, \star, s)$ at $s=k / 2$, and the value of the first non-vanishing derivative of $L_{p}(f, \Psi, \star, s)$, do not depend on the choice of $c$.

Because of this lemma, it is customary to drop the $c$ from the notations and write $L_{p}(f, \Psi, s)$ instead of $L_{p}(f, \Psi, \star, s)$.

If $\Psi_{1} \ldots, \Psi_{h}$ are representatives for the distinct classes of oriented optimal embeddings in $\operatorname{emb}(\mathcal{O}, R)$, note that

$$
L_{p}(f, K, k / 2)=L_{p}\left(f, \Psi_{1}, k / 2\right)+\cdots+L_{p}\left(f, \Psi_{h}, k / 2\right) .
$$

The following proposition describes the value of $L_{p}(f, K, s)$ and $L_{p}(f, \Psi, s)$ at the central critical point $s=k / 2$.

Proposition 2.22 Suppose $p$ is split in $K$. 
1. If $w=-1$, then

$$
L_{p}(f, K, k / 2)^{2}=4 u_{K}^{2} D^{\frac{k-1}{2}} \frac{L(\phi / K, k / 2)}{(\phi, \phi)} .
$$

2. If $w=1$, then $L_{p}(f, K, k / 2)=0$, and in fact $L_{p}(f, \Psi, k / 2)=0$ for all $\Psi \in \operatorname{emb}(\mathcal{O}, R)$.

Proof: Choose a vertex $v$ of $\mathcal{T}$ which is even and fixed under the action of $\iota \Psi\left(\mathcal{O}_{K} \otimes \mathbb{Z}_{p}\right)^{\times}$, and let $\delta=\iota \Psi(u)$. A direct evaluation shows that

$$
L_{p}(f, \Psi, k / 2)= \pm(1-w) \sum_{v \rightarrow \delta v} w^{s g n(e)} c_{f}(e)\left(P_{\Psi}^{\frac{k-2}{2}}\right)
$$

The result follows directly, using (22) and proposition 2.18 .

Proposition 2.23 Suppose $p$ is inert in $K$. Then $L_{p}(f, K, k / 2)=0$, and in fact $L_{p}(f, \Psi, k / 2)=0$ for all $\Psi \in \operatorname{emb}(\mathcal{O}, R)$.

Proof: This is apparent from the harmonicity of $c_{f}$ and the geometry of the action of $K_{p}^{\times} / \mathbb{Q}_{p}^{\times}$on $\mathcal{T}$ induced by $\Psi$.

Let $\Psi_{1}, \ldots, \Psi_{h}$ be representatives for the distinct classes in $\operatorname{emb}(\mathcal{O}, R)$. In the cases where $p$ is inert in $K$ or where $p$ is split and $w=1$, so that $L_{p}\left(f, \Psi_{i}, k / 2\right)=0$ for all $i$, the first derivative of the $p$-adic $L$-function $L_{p}(f, K, s)$ at $s=k / 2$ is given by:

$$
L_{p}^{\prime}(f, K, k / 2)=\int_{\tilde{G}} \log (\alpha) d \mu_{f, K}(\alpha)=\sum_{i=1}^{h} L_{p}^{\prime}\left(f, \Psi_{i}, k / 2\right),
$$

and

$$
L_{p}^{\prime}\left(f, \Psi_{i}, k / 2\right)=\int_{G} \log (\alpha) d \mu_{f, \Psi_{i}}(\alpha)
$$

These formulas are consequences of the continuity of $\mu_{f, \Psi_{i}}$ for all $i$. The goal of the next chapter is to derive a formula for these first derivatives. 


\section{Proof of the main identities}

Sections 3.1 and 3.2 contain the proofs of the main identities in the case where $p$ is split in $K$. In this situation an exceptional zero arises only if $w=1$, which will be assumed from now on for those sections. Section 3.3 discusses the case when $p$ is inert in $K$.

\subsection{Teitelbaum's $\mathcal{L}$-invariant}

The definition of Teitelbaum's $\mathcal{L}$-invariant attached to $f$ is recalled in this section. It depends on the choice of $p$-adic logarithm that was made in section 2.6 .

First let us point out that the modular form $f$ is actually a modular form for the group $\tilde{\Gamma}$, as a consequence of the assumption $w=1$. Choose a point $z_{0} \in \mathcal{H}_{p}$, and a vertex $v \in \mathcal{T}$.

Proposition 3.1 (Teitelbaum) There exists $\mathcal{L}_{T}(f) \in \mathbb{C}_{p}$ and $R \in \mathcal{P}_{k-2}^{\vee}$ (the latter, depending on the choice of $z_{0}$ and $v$ ) such that, for all $\alpha \in \tilde{\Gamma}$ and $r \in \mathcal{P}_{k-2}$,

$$
\int_{z_{0}}^{\alpha * z_{0}} f(z) r(z) d z=\mathcal{L}_{T}(f) \cdot \sum_{v \rightarrow \alpha v} c_{f}(e)(r)+(\alpha \cdot R(r)-R(r)) .
$$

Here the integral on the left is Coleman's integral associated to the choice of $\log$, and the sum on the right is taken over the edges in the path joining $v$ to $\alpha v$.

The proof is given in [Tei], sec. 1 .

Definition 3.2 The scalar $\mathcal{L}_{T}(f)$ (which depends only on the homothety class of $f$ ) is called the Teitelbaum $\mathcal{L}$-invariant associated to $f$.

The coboundary term $(\alpha R(r)-R(r))$, which vanishes when $k=2$, presents an extra difficulty in computing $\mathcal{L}_{T}(f)$ in the higher weight case. To avoid this extra term, let $\delta$ be any element of $\tilde{\Gamma}$, let $\psi=\delta-\frac{1}{2} \operatorname{trace}(\delta)\left(\begin{array}{ll}1 & 0 \\ 0 & 1\end{array}\right) \in$ $M_{2}\left(\mathbb{Q}_{p}\right)$, and write $P_{\psi}$ for the polynomial $P_{\iota^{-1}(\psi)} \in \mathcal{P}_{2}$ defined by formula (3). Note that $P_{\psi} \cdot \delta=P_{\psi}$, and the same invariance property holds for 
$P_{\psi}^{\frac{k-2}{2}} \in \mathcal{P}_{k-2}$. Replacing $\alpha$ by $\delta$ and $r$ by $P_{\psi}^{\frac{k-2}{2}}$ yields a formula which does not involve any coboundary term and determines $\mathcal{L}_{T}(f)$ when the sum on the right is non-zero:

$$
\int_{z_{0}}^{\delta * z_{0}} f(z) P_{\psi}^{\frac{k-2}{2}}(z) d z=\mathcal{L}_{T}(f) \sum_{v \rightarrow \delta v} c_{f}(e)\left(P_{\psi}^{\frac{k-2}{2}}\right) .
$$

Note that both the integral and the sum in the above equation are independent of the choice of base points $z_{0}$ and $v$ respectively.

\subsection{The split case}

In this section suppose that $p$ is split in $K$, and let $\mathfrak{p}$ be a prime of $K$ above $p$. Let us recall the notations of section 1.3, i.e. let $u_{0}$ denote a generator of the rank one group $\mathcal{O}^{\times} / \mathbb{Z}[1 / p]^{\times}$such that the image $u$ of $\frac{u_{0}}{\bar{u}_{0}}$ in $\mathbb{Q}_{p}^{\times}$has the property $\operatorname{ord}_{p}(u)>0$. If $\Psi$ is a representative for an element of $\operatorname{emb}(\mathcal{O}, R)$, write $\delta:=\iota \Psi\left(u_{0}\right) \in \tilde{\Gamma}$. (This gives rise to the $p$-adic Shintani cycle attached to $\Psi$, as in the discussion following proposition 2.18.) Let $v$ be an even vertex of $\mathcal{T}$ which is fixed by $\iota \Psi\left(\mathcal{O}_{K} \otimes \mathbb{Z}_{p}\right)^{\times}$.

\section{Theorem 3.3}

$$
L_{p}^{\prime}(f, \Psi, k / 2)=\mathcal{L}_{T}(f) \sum_{v \rightarrow \delta v} c_{f}(e)\left(P_{\Psi}^{\frac{k-2}{2}}\right)
$$

where the sum on the right is taken over all edges e in the path joining $v$ to $\delta v$.

Proof: Consider the left-hand side of (25) with this choice of $\delta$, and note that the polynomial denoted there by $P_{\psi}$ is a constant multiple of $P_{\Psi}$.

By Teitelbaum's $p$-adic Poisson inversion formula (proposition 1.11),

$$
I:=\int_{z_{0}}^{\delta * z_{0}} f(z) P_{\Psi}^{\frac{k-2}{2}}(z) d z=\int_{z_{0}}^{\delta * z_{0}}\left(\int_{\mathbb{P}_{1}\left(\mathbb{Q}_{p}\right)} \frac{1}{z-x} d \mu_{f}(x)\right) P_{\Psi}^{\frac{k-2}{2}}(z) d z .
$$

Observe that

$$
\frac{P_{\Psi}^{\frac{k-2}{2}}(z)-P_{\Psi}^{\frac{k-2}{2}}(x)}{z-x}=\text { polynomial in } x \text { of degree } \leq k-2 .
$$


Hence, by lemma 1.9,

$$
\int_{\mathbb{P}_{1}\left(\mathbb{Q}_{p}\right)} \frac{P_{\Psi}^{\frac{k-2}{2}}(z)}{z-x} d \mu_{f}(x)=\int_{\mathbb{P}_{1}\left(\mathbb{Q}_{p}\right)} \frac{P_{\Psi}^{\frac{k-2}{2}}(x)}{z-x} d \mu_{f}(x) .
$$

Therefore the polynomial $P_{\Psi}^{\frac{k-2}{2}}(z)$ can be replaced by $P_{\Psi}^{\frac{k-2}{2}}(x)$ in equation (26). Applying the reasoning in the proof of theorem 4 of [Tei], reverse the order of integration to obtain

$$
\begin{aligned}
I & =\int_{\mathbb{P}_{1}\left(\mathbb{Q}_{p}\right)}\left(\int_{z_{0}}^{\delta * z_{0}} \frac{d z}{z-x}\right) P_{\Psi}^{\frac{k-2}{2}}(x) d \mu_{f}(x) \\
& =\int_{\mathbb{P}_{1}\left(\mathbb{Q}_{p}\right)} \log \left(\frac{\delta * z_{0}-x}{z_{0}-x}\right) P_{\Psi}^{\frac{k-2}{2}}(x) d \mu_{f}(x),
\end{aligned}
$$

where the last equality follows from the definition of the Coleman integral associated to this choice of log. Recall the Möbius transformation $\eta_{\Psi}: \mathbb{P}_{1}\left(\mathbb{C}_{p}\right) \longrightarrow \mathbb{P}_{1}\left(\mathbb{C}_{p}\right)$ of section 2.4. Define $\beta_{0} \in \mathbb{P}_{1}\left(\mathbb{C}_{p}\right)$ by $\eta_{\Psi}\left(\beta_{0}\right)=z_{0}$. Performing the change of variables $x=\eta_{\Psi}(\alpha)$ and applying the definition of $\mu_{\Psi}=\mu_{f, \Psi, \star}$

$$
I=\int_{\mathbb{P}_{1}\left(\mathbb{Q}_{p}\right)} \log \left(\frac{u \beta_{0}-\alpha}{\beta_{0}-\alpha}\right) d \mu_{\Psi}(\alpha) .
$$

Note that if $\alpha=0$ or $\infty$, the integrand vanishes, because of the choice of logarithm that was made in the definition of the Coleman integral. Since $\log (u)=0$, observe that

$$
\begin{aligned}
\sum_{n=-\infty}^{\infty} \log \left(\frac{u \beta_{0}-u^{n} \alpha}{\beta_{0}-u^{n} \alpha}\right) & =\lim _{N \rightarrow \infty} \sum_{n=-N}^{N} \log \left(\frac{\beta_{0}-u^{n-1} \alpha}{\beta_{0}-u^{n} \alpha}\right) \\
=\lim _{N \rightarrow \infty} \log \left(\frac{\beta_{0}-u^{-N-1} \alpha}{\beta_{0}-u^{N} \alpha}\right) & =\lim _{N \rightarrow \infty} \log \left(\frac{u^{N+1} \beta_{0}-\alpha}{\beta_{0}-u^{N} \alpha}\right)=\log \alpha-\log \beta_{0} .
\end{aligned}
$$

Applying the proposition 2.12 it follows that

$$
I=\int_{G}\left(\sum_{n=-\infty}^{\infty} \log \frac{u \beta_{0}-u^{n} \alpha}{\beta_{0}-u^{n} \alpha}\right) d \mu_{\Psi}(\alpha)=\int_{G}\left(\log \alpha-\log \beta_{0}\right) d \mu_{\Psi}(\alpha) .
$$


Note that

$$
\int_{G} \log \left(\beta_{0}\right) d \mu_{\Psi}(\alpha)=\log \left(\beta_{0}\right) L_{p}(f, \Psi, k / 2)=0,
$$

by proposition $2.22,(2)$. It follows that

$$
I=\int_{G} \log (\alpha) d \mu_{\Psi}(\alpha)=L_{p}^{\prime}(f, \Psi, k / 2),
$$

by $(24)$. On the other hand, formula $(25)$ for Teitelbaum's $\mathcal{L}$-invariant shows that

$$
I=\mathcal{L}_{T}(f) \sum_{v \rightarrow \delta v} c_{f}(e)\left(P_{\Psi}^{\frac{k-2}{2}}\right)
$$

Theorem 3.3 follows from (31) and (32).

The following theorem can be viewed as the analogue of Teitelbaum's conjecture in the anticyclotomic setting.

Theorem 3.4 Assume conjecture 2.17. Then

$$
L_{p}^{\prime}(f, K, k / 2)^{2}=\mathcal{L}_{T}(f)^{2} u_{K}^{2} D_{K}^{\frac{k-1}{2}} \frac{L(\phi / K, k / 2)}{(\phi, \phi)} .
$$

Proof: Let $\Psi_{1}, \ldots, \Psi_{h}$ be distinct representatives for the oriented optimal embeddings in $\operatorname{emb}(\mathcal{O}, R)$. For $1 \leq j \leq h$, choose even vertices $v_{j}$ of $\mathcal{T}$ which are fixed by $\iota \Psi_{j}\left(\left(\mathcal{O}_{K} \otimes \mathbb{Z}_{p}\right)^{\times}\right)$and write $\delta_{j}=\iota \Psi_{j}\left(u_{0}\right)$. By formula $(23)$ combined with theorem 3.3 ,

$$
L_{p}^{\prime}(f, K, k / 2)=\mathcal{L}_{T}(f) \sum_{j=1}^{h} \sum_{v_{j} \rightarrow \delta_{j} v_{j}} c_{f}(e)\left(P_{\Psi_{j}}^{\frac{k-2}{2}}\right) .
$$

Theorem 3.4 now follows from proposition 2.18.

Remark: In [Kl] Klingenberg claims to prove an "exceptional zero conjecture", analogous to theorem 3.3, for Schneider's rigid analytic $p$-adic $L$ function. Klingenberg normalizes the Schneider $L$-function by choosing a special isomorphism $B_{p} \rightarrow M_{2}\left(\mathbb{Q}_{p}\right)$; this depends on the choice of a normalization datum ([Kl], definition 6.3.1) which need not exist in general. Indeed, assume such a datum to be given. Then there is a maximal $\mathbb{Z}$-order $\mathcal{O}^{\prime}$ in 
$B$ and an element $\delta_{0} \in \mathcal{O}^{\prime}[1 / p]^{\times}$such that for the zeros $\alpha, \beta \in \mathbb{Q}_{p}$ of the minimal polynomial of $\delta_{0} / \mathbb{Q}$ we have $\operatorname{ord}(\alpha / \beta)=1$. After multiplying $\delta_{0}$ by a suitable power of $p$ one can assume that $\delta_{0}$ belongs to $\mathcal{O}^{\prime}$ and that its reduced norm is $p$. Set $K=\mathbb{Q}\left(\delta_{0}\right)$. It is an imaginary quadratic field and the minimal polynomial of $\delta_{0}$ over $\mathbb{Q}$ is of the form

$$
f(X)=X^{2}+a X+p \quad \text { with } a^{2}<4 p .
$$

There are only finitely many such fields and hence there exist quaternion algebras $B$ which do not contain any of them and thus do not admit a normalization datum. (For example, choose $B$ such that its discriminant has a prime factor $q$ which splits in all the fields $K$ as above.)

In addition, theorem 4.6.1 and 4.6.2 of [Kl] are not correct as stated. In fact the proofs of these theorems actually yield the following (adopting the notation from $[\mathrm{Kl}])$ :

Let $\gamma, \delta \in \Gamma$ be such that $[\gamma]$ and $[\delta] \in \Gamma^{a b}$ are non-zero and assume that $L_{p}\left(c_{\gamma}, 1\right)=0$. Assume moreover that $\delta$ is hyperbolic. Then for every homomorphism $\chi \in \operatorname{Hom}\left(\mathbb{Q}_{p}^{\times}, \mathbb{Q}_{p}\right)$ the following holds

$$
\int_{\mathbb{Z}_{p}[\delta]} \chi(x) d \mu_{\gamma}(x)=\chi\left(\langle\gamma, \delta\rangle_{\Gamma} \operatorname{mult}(\delta)^{m}\right)
$$

Here $m$ is the unique integer such that $\langle\gamma, \delta\rangle_{\Gamma}$ mult $(\delta)^{m} \in \mathbb{Z}_{p}[\delta]$.

Consequently, theorems 6.4.2 and corollary 6.4.1 of [Kl] do not hold as stated. (A summand $\log _{p}\left(\operatorname{mult}(\delta)^{m}\right)$ is missing on the right hand side of the equations there and it is usually non-zero.)

While it seems unlikely that there is any simple relation between Schneider's $p$-adic $L$-function (with respect to a suitable normalization) and the cyclotomic $p$-adic $L$-function of Mazur, Tate and Teitelbaum as is speculated in $([\mathrm{Kl}], \mathrm{p} .313)$, the arguments of $[\mathrm{Kl}]$ can be adapted to give a proof of theorem 3.3 in the case of weight 2 . Together with the result on the interpolation of classical special values of section 2.5, this yields a proof of the exceptional zero conjecture for the anticyclotomic $p$-adic $L$-function when $k=2$.

We sketch briefly now how one can deduce theorem 3.3 in the weight two case from (33). For simplicity assume that $K$ has class number one. Then $\operatorname{ord}_{\mathfrak{p}}(u)=1$ and $(33)$ gives

$$
L_{p}^{\prime}(f, K, 1)=\int_{\mathbb{Z}_{p}^{\times}} \log (x) d \mu_{f}(x)=\log \left(\left\langle\delta, c_{f}\right\rangle\right),
$$


since $\operatorname{mult}(\delta)=u^{2}$ and thus $\log (\operatorname{mult}(\delta))=0$. Now $([\mathrm{Kl}], 5.3$ and proposition 3.3.1) imply that

$$
L_{p}^{\prime}(f, K, 1)=\log \left(\left\langle\delta, c_{f}\right\rangle\right) \sum_{v \rightarrow \delta v} c_{f}(e)
$$

\subsection{The inert case}

Assume now that $p$ is inert in $K$.

Theorem 3.5 Let $z_{0}$ and $\bar{z}_{0} \in \mathcal{H}_{p}$ be the two fixed points for $\iota \Psi\left(K_{p}^{\times}\right)$acting on $\mathcal{H}_{p}$. Then

$$
L_{p}^{\prime}(f, \Psi, k / 2)= \pm \int_{\bar{z}_{0}}^{z_{0}} f(z) P_{\Psi}^{\frac{k-2}{2}}(z) d z .
$$

Proof: Beginning with the right hand side of theorem 3.5,

$$
I:=\int_{\bar{z}_{0}}^{z_{0}} f(z) P_{\Psi}^{\frac{k-2}{2}}(z) d z=\int_{\bar{z}_{0}}^{z_{0}}\left(\int_{\mathbb{P}_{1}\left(\mathbb{Q}_{p}\right)} \frac{1}{z-x} d \mu_{f}(x)\right) P_{\Psi}^{\frac{k-2}{2}}(z) d z,
$$

by Teitelbaum's p-adic Poisson inversion formula (proposition 1.11). Now observe that

$$
\frac{P_{\Psi}^{\frac{k-2}{2}}(z)-P_{\Psi}^{\frac{k-2}{2}}(x)}{z-x}=\text { polynomial in } x \text { of degree } \leq k-2 .
$$

Hence, by lemma 1.9,

$$
\int_{\mathbb{P}_{1}\left(\mathbb{Q}_{p}\right)} \frac{P_{\Psi}^{\frac{k-2}{2}}(z)}{z-x} d \mu_{f}(x)=\int_{\mathbb{P}_{1}\left(\mathbb{Q}_{p}\right)} \frac{P_{\Psi}^{\frac{k-2}{2}}(x)}{z-x} d \mu_{f}(x) .
$$

Therefore the polynomial $P_{\Psi}^{\frac{k-2}{2}}(z)$ can be replaced by $P_{\Psi}^{\frac{k-2}{2}}(x)$ in equation (34). Reversing the order of integration as in (27),

$$
\begin{aligned}
I & =\int_{\mathbb{P}_{1}\left(\mathbb{Q}_{p}\right)}\left(\int_{\bar{z}_{0}}^{z_{0}} \frac{d z}{z-x}\right) P_{\Psi}^{\frac{k-2}{2}}(x) d \mu_{f}(x) \\
& =\int_{\mathbb{P}_{1}\left(\mathbb{Q}_{p}\right)} \log \left(\frac{x-z_{0}}{x-\bar{z}_{0}}\right) P_{\Psi}^{\frac{k-2}{2}}(x) d \mu_{f}(x) \\
& =\int_{G} \log (\alpha) d \mu_{\Psi}(\alpha),
\end{aligned}
$$


where the last identity follows by making the change of variables $x=\eta_{\Psi}(\alpha)$ (cf. equation (15)). Hence

$$
I=L_{p}^{\prime}(f, \Psi, k / 2)
$$

by (24). The result follows.

Remark: By the Cerednik-Drinfeld theorem, the rigid analytic curve $\mathcal{H}_{p} / \Gamma$ has a model over $\mathbb{Q}$ given by a Shimura curve $X$ classifying abelian surfaces with quaternionic multiplication and auxiliary level structure. (Cf. [BD3], $\S 4$.) Using Drinfeld's moduli interpretation of $\mathcal{H}_{p}$, it is shown in section 5 of [BD3] that the points $z_{0}$ and $\bar{z}_{0}$ correspond to CM points on $X$ defined over the Hilbert class field $H$ of $K$. Assuming $k=2$, the modular form $f$ corresponds to a quotient $A_{f}$ defined over $\mathbb{Q}$ of the Jacobian $J$ of $X$; in this situation, the integral appearing on the right hand side of the identity of theorem 3.5 is related to the natural image of the divisor $\left(z_{0}\right)-\left(\bar{z}_{0}\right)$ in $A_{f}(H)$ via the $p$-adic Abel-Jacobi map. One recovers the main result of [BD3], which gives a construction, in terms the first derivative of the anticyclotomic $p$-adic $L$-function, of a Heegner point on $A_{f}(K)$ and implies that this point is of infinite order when $L_{p}^{\prime}(f, K, 1) \neq 0$.

For general $k$, the integral appearing in theorem 3.5 can be interpreted as the image by a higher $p$-adic Abel-Jacobi map of certain CM cycles in the Chow groups of the Kuga-Sato variety attached to forms of weight $k$. This interpretation will be explained in a future work.

\section{References}

[BD1] M. Bertolini and H. Darmon, Heegner points on Mumford-Tate curves, Invent. Math. 126 (1996) 413-456.

[BD2] M. Bertolini and H. Darmon (with an appendix by B. Edixhoven) A rigid analytic Gross-Zagier formula and arithmetic applications, Ann. of Math. (2) 146 (1997) 111-147.

[BD3] M. Bertolini and H. Darmon, Heegner points, p-adic L-functions and the Cerednik-Drinfeld uniformisation, Invent. Math. 131 (1998) 453-491. 
[BD4] M. Bertolini and H. Darmon, p-adic periods, p-adic L-functions and the p-adic uniformisation of Shimura curves, Duke Math J. 98 (1999) 305-334.

[BD5] M. Bertolini and H. Darmon, The p-adic L-functions of modular elliptic curves. To appear in 2001 and Beyond, Springer Verlag.

[BC] J-F. Boutot and H. Carayol, Uniformisation p-adique des courbes de Shimura: les théorèmes de Cerednik et de Drinfeld. Courbes modulaires et courbes de Shimura (Orsay, 1987/1988). Astérisque No. 196-197 7 (1991) 45-158.

[Ce] I.V. Cerednik, Uniformisation of algebraic curves by discrete arithmetic subgroups of $\mathrm{PGL}_{2}\left(k_{w}\right)$ with compact quotient spaces. (Russian) Mat. Sb. (N.S.) 100 (1976) 59-88.

[CI] R.F. Coleman and A. Iovita, Frobenius and monodromy operators for p-adic de Rham cohomology via degeneration, in preparation.

[Co] R.F. Coleman, A p-adic Shimura isomorphism and p-adic periods of modular forms. $p$-adic monodromy and the Birch and SwinnertonDyer conjecture (Boston, MA, 1991), Contemp. Math. 165 (1994) $21-51$.

[Dag] H. Daghigh, Quaternion algebras, modular forms, and special values of $L$-series, McGill PhD thesis, 1997.

[Da] H. Darmon, Integration on $\mathcal{H}_{p} \times \mathcal{H}$ and arithmetic applications, Annals of Mathematics, to appear.

[Dr] Drinfeld, V. G. Coverings of p-adic symmetric domains. (Russian) Funkcional. Anal. i Priložen. 10 (1976) 29-40.

[GvdP] L. Gerritzen, M. van der Put, Schottky Groups and Mumford Curves, Springer Lecture Notes 8171980.

[GS] R. Greenberg and G. Stevens, p-adic L-functions and p-adic periods of modular forms, Invent. Math. 111 (1993) 407-447.

[Gr1] B.H. Gross. Heegner points on $X_{0}(N)$. In Modular forms (Durham, 1983), Ellis Horwood Ser. Math. Appl.: Statist. Oper. Res., Horwood, Chichester (1984) 87-105.

[Gr2] B.H. Gross. Heights and the special values of L-series. Number theory (Montreal, Que., 1985) CMS Conf. Proc. 7 (1987) 115-187. 
[Ha] R.L. Hatcher, Heights and L-series. Canad. J. Math. 42 (1990) $533-560$.

[JL1] B.W. Jordan and R. Livne, Local diophantine properties of Shimura curves, Math. Ann. 270 (1985) 235-248.

[JL2] B.W. Jordan and R. Livne, Integral Hodge theory and congruences between modular forms, Duke Math. J. 80 (1995) 419-484.

[Kl] C. Klingenberg, On p-adic L-functions of Mumford curves, in " $p$ adic Monodromy and the Birch and Swinnerton-Dyer conjecture", Contemp Math 165 (1994) 277-315.

[MTT] B. Mazur, J. Tate, and J. Teitelbaum, On p-adic analogues of the conjectures of Birch and Swinnerton-Dyer, Invent. Math. 84 (1986) $1-48$.

[Mz1] B. Mazur, On monodromy invariants occurring in global arithmetic, and Fontaine's theory. p-adic monodromy and the Birch and Swinnerton-Dyer conjecture (Boston, MA, 1991), Contemp. Math. 165 (1994) 1-20.

[PR] B. Perrin-Riou, Fonctions L p-adiques associées à une forme modulaire et à un corps quadratique imaginaire. J. London Math. Soc. (2) 38 (1988) 1-32.

[Rob] D. Roberts. Shimura curves analogous to $X_{0}(N)$. Harvard PhD thesis, 1989 .

[Sch] P. Schneider, Rigid-analytic L-transforms. Number theory, Noordwijkerhout 1983, Lecture Notes in Math. 1068 (1984) 216-230.

[SS] P. Schneider, U. Stuhler, The cohomology of p-adic symmetric spaces, Invent.Math. 105 (1991) 47-122.

[St] G. Stevens, Overconvergent Modular Symbols, (in preparation).

[Tei] J.T. Teitelbaum, Values of p-adic L-functions and a p-adic Poisson kernel, Invent. Math. 101 (1990) 395-410.

[Va] N. Vatsal, manuscript, to appear.

[Vi] M-F. Vignéras, Arithmétique des algèbres de quaternions. Lecture Notes in Mathematics 800. Springer, Berlin, 1980. 